\title{
THE STRUCTURE OF PSEUDO-INVERSE SEMIGROUPS
}

\author{
BY \\ F. PASTIJN ${ }^{1}$
}

\begin{abstract}
A regular semigroup $S$ is called a pseudo-inverse semigroup if $e S e$ is an inverse semigroup for each $e=e^{2} \in S$. We show that every pseudo-inverse semigroup divides a semidirect product of a completely simple semigroup and a semilattice. We thereby give a structure theorem for pseudo-inverse semigroups in terms of groups, semilattices and morphisms. The structure theorem which is presented here generalizes several structure theorems which have been given for particular classes of pseudo-inverse semigroups by several authors, and thus contributes to a unification of the theory.
\end{abstract}

Completely (0-) simple semigroups and inverse semigroups form the first prototypes for the study of pseudo-inverse semigroups. We therefore can say that the theory of regular semigroups began with the study of pseudo-inverse semigroups [40, 45].

We may distinguish four successful trends in the papers which since then have dealt with some wider classes of pseudo-inverse semigroups: 1 . the subdirect products of completely 0 -simple and completely simple semigroups, 2 . the generalized inverse semigroups (orthodox pseudo-inverse semigroups, 3. the normal band compositions of inverse semigroups, and 4. Rees matrix semigroups over inverse semigroups (with zero).

Subdirect products of completely 0 -simple semigroups and completely simple semigroups were initiated in [13, Chapter 2] and studied in great detail in [18] (see also $\S 4$ of [14]); this class contains several interesting subclasses: (a) the trees of completely 0 -simple semigroups $[\mathbf{1 8}]$ which include the primitive regular semigroups [7, Vol. II, 16, 39, 44, 46], (b) the regular locally testable semigroups [50] which include the normal bands [36] and the combinatorial completely 0 -simple semigroups, (c) the normal bands of groups [37] which include the semilattices of groups [7, Vol. I], (d) the subdirect products of Brandt semigroups which include the locally testable semigroups which are inverse semigroups [50] and the primitive inverse semigroups [39]. The generalized inverse semigroups were introduced in [48] as a special class of orthodox semigroups; they include (a) the inverse semigroups, (b) the orthodox completely 0 -simple semigroups [9] and the rectangular groups, (c) the

Received by the editors October 25, 1979 and, in revised form, March 25, 1981 and June 18, 1981.

AMS (MOS) subject classifications (1970). Primary 20M10.

Key words and phrases. Pseudo-inverse semigroup, proper inverse semigroup, rectangular band of inverse semigroups, pseudo-semilattice, semilattice, order automorphism.

'The author's research was done while he was a visiting professor at the University of Nebraska, supported by a Fulbright-Hays Award. 
various classes of $L$ - [R-] unipotent pseudo-inverse semigroups [35], (d) the various classes of orthodox normal bands of inverse semigroups [33, 35, 42], in particular the orthodox normal bands of groups. The normal band compositions of inverse semigroups occur as special cases of more general considerations $[33,35,38$, Chapters 3 and 4, 42]; rectangular bands of inverse semigroups have been investigated in [35]; several authors consider semilattice compositions of particular inverse semigroups $[8,10,49]$ which of course yield special inverse semigroups. The idea of a Rees matrix representation has been exploited and applied to produce numerous classes of sophisticated semigroups; we refer the reader to [17, 35, §4], for the peculiar pseudo-inverse semigroups which have a structure theorem of Rees type.

The above considered classes of pseudo-inverse semigroups may overlap. However, so far no attempt has been made to establish a comprehensive classification.

Independently from the above-mentioned cases several devices have been invented to build pseudo-inverse semigroups [1, Chapter 4, 2, 19, 25, 47...]. Some recent papers concentrate on idempotent-generated nonprimitive pseudo-inverse semigroups $[2,3,4,15,24,34]$, and in [32] a countably infinite set of pairwise nonisomorphic bisimple nonprimitive pseudo-inverse semigroups with 3 idempotent generators has been constructed.

The class of pseudo-inverse semigroups was introduced in [29 and 30] as an overall generalization of the specific classes listed above. The structure theorem for pseudo-inverse semigroups, which is given in [29], presupposes the knowledge of the biordered set, the structure mappings and the trace products. The structure theorem which will be given in this paper follows a far different approach and is the result of a detailed investigation of the pseudo-semilattices as presented in [24].

1. Introduction and preliminary results. We assume that the reader is familiar with the standard notation and terminology of semigroup theory as established in 77 and 11]. We also assume that the reader is acquainted with the basic results by Nambooripad concerning biordered sets, the fundamental representation of a regular semigroup, and the fundamental regular semigroup $T_{E}$ which is associated with the biordered set $\left(E, \omega^{l}, \omega^{r}, \tau\right)[5,6,26,27,28]$. The notation and terminology which is listed below is in accordance with [24] and slightly modifies Nambooripad's terminology of [ 29 and 30].

A regular semigroup $S$ is called a pseudo-inverse semigroup if, for all $e=e^{2} \in S$, $e S e$ is an inverse semigroup. The class of pseudo-inverse semigroups is closed for taking regular subsemigroups, homomorphic images and direct products [30].

Let $S$ be a regular semigroup. The set of idempotents of $S$ will be denoted by $E(S)$. We introduce the relations $\omega^{l}$ and $\omega^{r}$ on $E(S)$ in the following way: if $e, f \in E(S)$, then we put $e \omega^{l} f\left[e \omega^{r} f\right]$ if and only if $e=e f[e=f e]$ in $S$. The relations $\omega^{l}$ and $\omega^{r}$ are quasi-orders, the relation $\omega=\omega^{l} \cap \omega^{r}$ is a partial order, and the relations $\omega^{l} \cap\left(\omega^{l}\right)^{-1}, \omega^{r} \cap\left(\omega^{r}\right)^{-1}$ are equivalence relations. If $S$ is a pseudo-inverse semigroup, then $\left(E(S), \omega^{l}, \omega^{r}\right)$ will be called a pseudo-semilattice. Pseudo-semilattices were introduced in [29 and 41]. Pseudo-semilattices have been characterized axiomatically in [29], and they were constructed in terms of semilattices and semilattice-morphisms in [24]. 
The natural partial order $\leqslant$ on a regular semigroup $S$ was introduced and investigated in [30]. If $x$ and $y$ are elements of the regular semigroup $S$, then we put $x \leqslant y$ if and only if

(1.1) $R_{x} \leqslant R_{y}$ and for some (all) $e=e^{2} \in R_{y}$ there exists $f=f^{2} \in R_{x}$ such that fwe and $x=f y$.

One can show that this definition is self-dual [30]. Remark that $\omega$ is the restriction of $\leqslant$ to $E(S)$. A subset $A$ of $S$ will be called an order-ideal of $S$ if, for all $y \in S$, $x \in A, y \leqslant x$ implies that $y \in A$.

Let $S$ be a regular semigroup, and suppose that $e, f, g \in E(S)$, with $e \omega^{r} g, f \omega^{l} g$. The mappings

$$
\begin{array}{ll}
\phi_{g, e}: R_{g} \rightarrow R_{e}, & x \rightarrow e x, \\
\psi_{g, f}: L_{g} \rightarrow L_{f}, & y \rightarrow y f,
\end{array}
$$

are called structure mappings of $S$ [26] (see also [23]). Remark that for all $x \in R_{g}$ and for all $y \in L_{g}$ we have $x \phi_{g, e} \leqslant x$ and $y \psi_{g, f} \leqslant y$.

We are now in the position to state Nambooripad's alternative characterizations of pseudo-inverse semigroups [28, 29].

RESULT 1. Let $S$ be a regular semigroup. The following conditions are equivalent:

(i) For all $e \in E(S)$, eSe is an inverse semigroup (i.e. $S$ is a pseudo-inverse semigroup).

(ii) For all $e \in E(S), \omega(e)$ is a semilatice.

(iii) For all $e \in E(S), \omega^{l}(e)$ forms a left normal band and $\omega^{r}(e)$ forms a right normal band.

(iv) The natural partial order $\leqslant$ on $S$ is compatible with the multiplication.

(v) For all $e, f \in E(S)$ there exists an element of $e \wedge f \in E(S)$ such that $\omega^{r}(e) \cap$ $\omega^{l}(f)=\omega(e \wedge f)$.

(vi) If $e, f, g \in E(S)$ and $e \Re f, e, f \in \omega(g)$, then $e=f$; if $e, f, g \in E(S)$ and

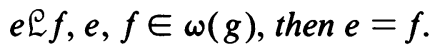

(vii) If $e, f, g, h \in E(S)$ and $e \Re f \omega^{r} g \Re h$, then $\phi_{g, e}=\phi_{h, f}$; if $e, f, g, h \in E(S)$ and $e \mathcal{L} f \omega^{l} g \mathcal{L} h$, then $\psi_{g, e}=\psi_{h, f}$.

Let $S$ be a pseudo-inverse semigroup, and let us suppose that $e, f \in E(S), e \omega^{r} f$. From Result 1 it follows that for every $y \in R_{f}$ there exists a unique $x \in R_{e}$ such that $x \leqslant y$ : this element $x$ is given by $x=e y=y \phi_{f, e}$. Furthermore, the structure mapping which maps $R_{y}$ into $R_{x}$ is unique, and independent of the choice of $f$ in $R_{y}$ and $e$ in $R_{x}$. A dual statement applies for structure mappings which map $\mathcal{L}$-classes into b-classes.

Let $\left(E, \omega^{l}, \omega^{r}\right)$ be any pseudo-semilattice. For every $e, f \in E$ define $e \wedge f$ by Result $1(\mathrm{v}) ; E, \wedge$ then becomes an idempotent binary algebra. We shall say that the pseudo-semilattice $\left(E^{\prime}, \omega^{l}, \omega^{r}\right)$ is an order-ideal of the pseudo-semilattice $\left(E, \omega^{l}, \omega^{r}\right)$ if $E^{\prime}, \wedge$ is a subalgebra of $E, \wedge$, and if for every $f \in E, e \in E^{\prime}$, fwe implies that $f \in E^{\prime}$.

If $\left(E, \omega^{l}, \omega^{r}\right)$ is a pseudo-semilattice, then there exists a unique family $\tau$ of partial transformations of $E$ such that $\left(E, \omega^{l}, \omega^{r}, \tau\right)$ is a biordered set [29]. 
If $S$ is a regular semigroup and $x, y$ elements of $S$ such that there exists an

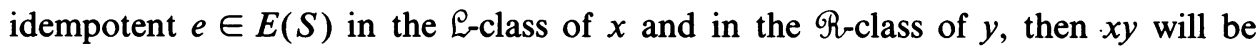
called a trace product of $x$ and $y$ in $S$. It follows from [7, Theorem 2.17], that $x y$ is a trace product of $x$ and $y$ in $S$ if and only if $x y \in R_{x} \cap L_{y}$. Let us now suppose that $S$ is a pseudo-inverse semigroup, and $e, f \in E(S)$. If $e \wedge f \in E(S)$ is the idempotent which is defined by Result $1(\mathrm{v})$, then $\{e \wedge f\}=S(f, e)$ constitutes the so-called sandwich set of $f$ and $e$ in $S$ [26]. Let $x, y \in S, e, f \in E(S)$, and suppose that $f \mathcal{L} x$ and $e \Re y$ in $S$; then

$$
x y=x(e \wedge f) y=(x(e \wedge f))((e \wedge f) y)=\left(x \psi_{f, e \wedge f}\right)\left(y \phi_{e, e \wedge f}\right)
$$

where $x(e \wedge f) \mathscr{L} e \wedge f \Re(e \wedge f) y$ and $x(e \wedge f) \leqslant x,(e \wedge f) y \leqslant y$ [26]. This implies that every product of elements of $S$ can be reduced to a trace product, and that the multiplication on $S$ is completely determined if we know the natural partial order $\leqslant$ and the trace products (see also [23]).

Let $\left(E, \omega^{l}, \omega^{r}\right)$ be a pseudo-semilattice. In [35, Theorem 4.25] it has been shown that $\left(E, \omega^{l}, \omega^{r}\right)$ is the pseudo-semilattice which is determined by some pseudo-inverse semigroup which has injective structure mappings if and only if $E$ is the disjoint union of its maximal subsemilattices (with respect to the partial order $\omega$ ). Theorem 2.10 of [4] and Theorem 3.4 of [35] show how such pseudo-semilattices may be constructed. In this paper we only need a construction for a pseudo-semilattice which is the disjoint union of its maximal subsemilattices all of which are semilattices with an identity. We proceed to give an outline of this construction.

Let $I, \Lambda$ be index sets. Let $\left(L_{\lambda}, \lambda \in \Lambda\right)$ and $\left(R_{i}, i \in I\right)$ be indexed families of semilattices. Let $\left(M_{i \lambda},(i, \lambda) \in I \times \Lambda\right)$ be an indexed family of pairwise disjoint semilattices such that for every $(i, \lambda) \in I \times \Lambda, M_{i \lambda}$ is a semilattice with identity $1_{i \lambda}$. Let $\phi_{i \lambda}: M_{i \lambda} \rightarrow L_{\lambda}, \psi_{i \lambda}: M_{i \lambda} \rightarrow R_{i},(i, \lambda) \in I \times \Lambda$, be monomorphisms such that

$\left(\mathrm{V}_{1}\right) M_{i \lambda} \phi_{i \lambda}$ is a principal ideal of $L_{\lambda}$ and $M_{i \lambda} \psi_{i \lambda}$ is a principal ideal of $R_{i}$ for all $(i, \lambda) \in I \times \Lambda$,

$\left(\mathrm{V}_{2}\right) L_{\lambda}=\cup_{j \in I} M_{j \lambda} \phi_{j \lambda}$ and $R_{i}=\cup_{\kappa \in \Lambda} M_{i \kappa} \psi_{i \kappa}$ for all $\lambda \in$ $\Lambda$ and all $i \in I$.

Let us put $E=\cup_{i \in I ; \lambda \in \Lambda} M_{i \lambda}$. On $E$ we define the relations $\omega^{l}$ and $\omega^{r}$ as follows. If $e_{i \lambda} \in M_{i \lambda}, f_{j \mu} \in M_{j \mu}, i, j \in I, \lambda, \mu \in \Lambda$, then we put

$$
\begin{aligned}
& e_{i \lambda} \omega^{r} f_{j \mu} \text { if and only if } i=j \text { and } e_{i \lambda} \psi_{i \lambda} \leqslant f_{i \mu} \psi_{i \mu} \text { in } R_{i}, \\
& e_{i \lambda} \omega^{l} f_{j \mu} \text { if and only if } \lambda=\mu \text { and } e_{i \lambda} \phi_{i \lambda} \leqslant f_{j \lambda} \phi_{j \lambda} \text { in } L_{\lambda} .
\end{aligned}
$$

This structure $\left(E, \omega^{l}, \omega^{r}\right)$ will henceforth be denoted by $E=\left(L_{\lambda}, R_{i} ; M_{i \lambda} ; \phi_{i \lambda}, \psi_{i \lambda}\right.$; $I, \Lambda)$.

Result 2. $E=\left(L_{\lambda}, R_{i} ; M_{i \lambda} ; \phi_{i \lambda}, \psi_{i \lambda} ; I, \Lambda\right)$ is a pseudo-semilattice which is the disjoint union of its maximal subsemilattices all of which are semilattices with an identity. Conversely, any such pseudo-semilattice can be so obtained.

It should not cause any confusion if we denote the meet operation in the semilattices $L_{\lambda}, R_{i}, M_{i \lambda},(i, \lambda) \in I \times \Lambda$, by $\wedge$. If we take $e_{i \lambda} \in M_{i \lambda}, f_{j \mu} \in M_{j \mu}$, then we define $e_{i \lambda} \wedge f_{j \mu}$ in $E$ by

$$
e_{i \lambda} \wedge f_{j \mu}=\left(e_{i \lambda} \psi_{i \lambda} \wedge 1_{i \mu} \psi_{i \mu}\right) \psi_{i \mu}^{-1} \wedge\left(f_{j \mu} \phi_{j \mu} \wedge 1_{i \mu} \phi_{i \mu}\right) \phi_{i \mu}^{-1}
$$


It is easy to check that this operation $\wedge$ on $E$ extends the meet operations which are given for the $M_{i \lambda},(i, \lambda) \in I \times \Lambda$, and that

$$
\omega^{r}\left(e_{i \lambda}\right) \cap \omega^{l}\left(f_{j \mu}\right)=\omega\left(e_{i \lambda} \wedge f_{j \mu}\right) .
$$

This is in accordance with Result $1(\mathrm{v})$.

Let us now suppose that $S$ is a pseudo-inverse semigroup and that $E=\left(L_{\lambda}, R_{i}\right.$; $\left.M_{i \lambda} ; \phi_{i \lambda}, \psi_{i \lambda} ; I, \Lambda\right)$ is the pseudo-semilattice which is determined by $S$. Let $(i, \lambda) \in I$ $\times \Lambda$, and suppose that $S_{i \lambda}$ is the subset of $S$ which consists of the elements $x$ for which there exist $j \in I, \mu \in \Lambda, e_{i \mu} \in M_{i \mu}, f_{j \lambda} \in M_{j \lambda}$ with $f_{j \lambda} \mathcal{L} x \Re e_{i \mu}$. We then have the following result.

Result 3. Let $S$ be a pseudo-inverse semigroup and let $E=\left(L_{\lambda}, R_{i} ; M_{i \lambda} ; \phi_{i \lambda}, \psi_{i \lambda}\right.$; $I, \Lambda)$ be the pseudo-semilattice which is determined by $S$. Then $S$ is a rectangular band $I \times \Lambda$ of the semigroups $S_{i \lambda},(i, \lambda) \in I \times \Lambda$. For every $(i, \lambda) \in I \times \Lambda$, we have $E\left(S_{i \lambda}\right)=M_{i \lambda}$, and $1_{i \lambda} S 1_{i \lambda}$ is the maximal inverse subsemigroup of $S_{i \lambda}$ which has $M_{i \lambda}$ as its semilattice of idempotents.

Proof. Anyhow $S=\cup_{i \in i ; \lambda \in \Lambda} S_{i \lambda}$ since $S$ is a regular semigroup. Let us now suppose that $x_{i \lambda} \in S_{i \lambda}$ and $y_{j \mu} \in S_{j \mu}$, and $x_{i \lambda} \Re e_{i \kappa}, y_{j \mu} \mathcal{L} f_{k \mu}$ for some $\kappa \in \Lambda, k \in I$, $e_{i \kappa} \in M_{i \kappa}, f_{k \mu} \in M_{k \mu}$. Let $x_{i \lambda} y_{j \mu} \in S_{n \nu}$ and $h_{s \nu} \mathcal{L} x_{i \lambda} y_{j \mu} R g_{n \sigma}$ for some $\sigma \in \Lambda, s \in I$, $g_{n \sigma} \in M_{n \sigma}, h_{s \nu} \in M_{s \nu}$. From $R_{x_{i \lambda} y_{j \mu}} \leqslant R_{x_{i \lambda}}$ and $L_{x_{i \lambda} y_{j \mu}} \leqslant L_{y_{j \mu}}$ then follows that $g_{n \sigma} \omega^{r} e_{i \kappa}$ and $h_{s \nu} \omega^{l} f_{k \mu}$. Consequently $i=n, \nu=\mu$ and $S_{n \nu}=S_{i \mu}$, and we conclude that $S_{i \lambda} S_{j \mu} \subseteq S_{i \mu}$. Thus $S$ is a rectangular band $I \times \Lambda$ of the semigroups $S_{i \lambda},(i, \lambda) \in I \times$ $\Lambda$. It is now clear that for every $(i, \lambda) \in I \times \Lambda, 1_{i \lambda} S 1_{i \lambda}$ is a subsemigroup of $S_{i \lambda}$ and that $E\left(S_{i \lambda}\right)=E\left(1_{i \lambda} S 1_{i \lambda}\right)=M_{i \lambda}$. From Result 1 we know that $1_{i \lambda} S 1_{i \lambda}$ is an inverse semigroup. Let $D$ be any inverse subsemigroup of $S$ for which $E(D)=M_{i \lambda}$. Since $1_{i \lambda}$ is the identity of $M_{i \lambda}, 1_{i \lambda}$ must be the identity for $D$ and so $D \subseteq 1_{i \lambda} S 1_{i \lambda}$. We conclude that $1_{i \lambda} S 1_{i \lambda}$ is the maximal inverse subsemigroup of $S_{i \lambda}$ which has $M_{i \lambda}$ as its semilattice of idempotents.

If $S$ is a pseudo-inverse semigroup and $E=\left(L_{\lambda}, R_{i} ; M_{i \lambda} ; \phi_{i \lambda}, \psi_{i \lambda} ; I, \Lambda\right)$ the pseudo-semilattice which is determined by $S$, then $I \times \Lambda$ is the greatest rectangular band homomorphic image of $S$, whereas the partitioning of $S$ into the subsemigroups $S_{i \lambda},(i, \lambda) \in I \times \Lambda$, determines the least rectangular band congruence on $S$. If $\gamma$ is a congruence on $S$ such that $S / \gamma$ is completely simple, then $\gamma$ will be called a primitive congruence on $S$. The least primitive congruence $\rho$ on $S$ must be contained in the least rectangular band congruence on $S$, and then $S / \rho$ may be identified with a Rees matrix semigroup $\mathfrak{N}(G ; P ; I, \Lambda)$. For every $(i, \lambda) \in I \times \Lambda, \rho$ induces a group congruence on $S_{i \lambda}$ and the quotient is precisely the maximal subgroup $G_{i \lambda}$ of TR $(G ; P ; I, \Lambda)$. In [30] Nambooripad gives a very convenient way to construct the least primitive congruence on the pseudo-inverse semigroup $S$ : if $\leqslant$ is the natural partial order on $S$, then the least primitive congruence $\rho$ on $S$ is given by $\leqslant \circ(\leqslant)^{-1}$. This is in accordance with the fact that, given any $x, y \in S$ with $x \leqslant y, x$ and $y$ belong to the same subsemigroup $S_{i \lambda}$ for some $(i, \lambda) \in I \times \Lambda$. The semigroup $S$ has injective structure mappings if and only if every $\rho$-class intersects every $R$-class and

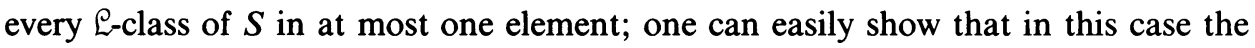
restriction of $\leqslant$ to a $\rho$-class structurizes this $\rho$-class to a semilattice (when considered as an ordered structure). For all $(i, \lambda) \in I \times \Lambda$, the semilattice $M_{i \lambda}$ is contained in a 
$\rho$-class, and $\rho^{\natural}$ maps all elements of $M_{i \lambda}$ onto the identity of the group $G_{i \lambda}$. It should be remarked that $M_{i \lambda}$ may be properly contained in a $\rho$-class, even in the case when $S$ has injective structure mappings. The following result will be useful for our future considerations.

RESUlT 4. Let $S$ be a pseudo-inverse semigroup, and let $E=\left(L_{\lambda}, R_{i} ; M_{i \lambda} ; \phi_{i \lambda}, \psi_{i \lambda}\right.$; $I, \Lambda)$ be the pseudo-semilattice which is determined by $S$. Then $S$ has injective structure mappings if and only if $S$ satisfies the following condition and its dual:

(1.6) if $(i, \lambda) \in I \times \Lambda, x_{i \lambda} \in S_{i \lambda}, e_{i \lambda}, f_{i \lambda} \in M_{i \lambda}, f_{i \lambda} \leqslant e_{i \lambda}, f_{i \lambda} \leqslant x_{i \lambda}$, and $e_{i \lambda} \& x_{i \lambda}$, then $e_{i \lambda}=x_{i \lambda}$.

A semigroup $S$ which is a rectangular band $I \times \Lambda$ of inverse semigroups $S_{i \lambda}$, $(i, \lambda) \in I \times \Lambda$, is a pseudo-inverse semigroup whose pseudo-semilattice of idempotents is the disjoint union of its maximal subsemilattices [35, Theorem 3.1, Corollary 3.2]. Such a semigroup will be called an elementary rectangular band of inverse semigroups if for all $(i, \lambda),(j, \mu) \in I \times \Lambda$ we have $S_{i \lambda} S_{j \mu}=S_{i \mu}$. A proper inverse semigroup is an inverse semigroup which has injective structure mappings [21, 22, 31]. $\$ 4$ of [35] gives a structure theorem for elementary rectangular bands of proper inverse semigroups. We summarize this structure theorem as follows.

RESUlt 5. Let $\mathcal{X}$ be a partially ordered set, and let $L$ be a subsemilattice and an ideal of $\mathcal{X}$. Let $G$ be a group which acts on $\mathcal{X}$ (on the left) as a group of order automorphisms. Let $I$ and $\Lambda$ be index sets, and for every $(i, \lambda) \in I \times \Lambda$, let $p_{\lambda i}$ be an element of $G$ such that the action of $p_{\lambda i}$ on $\mathcal{X}$ induces an order automorphism on L. Let $S$ be the set which consists of the elements $(A, g)_{i \lambda}, A \in L, g \in G, i \in I, \lambda \in \Lambda$, where $g^{-1} A \in L$. Define a multiplication on $S$ by

$$
(A, g)_{i \lambda}(B, h)_{j \mu}=\left(A \wedge g p_{\lambda j} B, g p_{\lambda j} h\right)_{i \mu} .
$$

$S$ then becomes an elementary rectangular band of proper inverse semigroups. Conversely, every elementary rectangular band of proper inverse semigroups can be so constructed.

The semigroup $S$ which is constructed in Result 5 will be denoted by $\mathscr{N}(P(G, \mathcal{X}, L) ; P ; I, \Lambda)$; in this notation $P$ is the $\Lambda \times I$-matrix which has the element $p_{\lambda i}$ in the $(\lambda, i)$-position. In case $|I|=|\Lambda|=1$, Result 5 reduces to McAlister's $P$-theorem which states that every proper inverse semigroup is isomorphic to some $P$-semigroup $P(G, X, L)$. We refer the reader to $[21,22,31]$ for more information concerning $P$-semigroups. In case $L=\mathscr{X}$ is trivial, Result 5 reduces to the well-known structure theorem for completely simple semigroups. The semigroup $\mathfrak{N}(P(G, \mathfrak{X}, L) ; P ; I, \Lambda)$ is a pseudo-inverse semigroup which has injective structure mappings. The maximal inverse subsemigroups of $\mathfrak{N}(P(G, \mathcal{X}, L) ; P ; I, \Lambda)$ are all isomorphic to the $P$-semigroup $P(G, \mathfrak{X}, L)$.

Any $P$-semigroup $P(G, L, L)$ is a semidirect product of a group and a semilattice, whereas a semigroup $\mathfrak{T}(P(G, L, L) ; P ; I, \Lambda)$ is a semidirect product of a completely simple semigroup and a semilattice. O'Carroll showed that every inverse semigroup divides an inverse semigroup of the form $P(G, L, L)$ [31]. One of our main results (see $\$ 4$ ) shows that every pseudo-inverse semigroup divides a pseudo-inverse semigroup of the form $\Re(P(G, L, L) ; P ; I, \Lambda)$. 
A congruence relation $\gamma$ on a regular semigroup $S$ will be called a strictly compatible congruence on $S$ if the $\gamma$-classes which contain idempotents form completely simple semigroups. Remark that every idempotent-separating congruence is a rather special strictly compatible congruence [12]. McAlister shows that every inverse semigroup is an idempotent-separating homomorphic image of an inverse semigroup which has injective structure mappings [22]. In our structure theorem for pseudo-inverse semigroups we shall show that, given any pseudo-inverse semigroup $S$, there exists a pseudo-inverse semigroup $\delta$ which has injective structure mappings, and a strictly compatible congruence $\gamma$ on $\delta$, such that $\delta / \gamma \cong S$; we shall construct such a pseudo-inverse semigroup $\delta$ in terms of groups, semilattices and morphisms. $\S \S 2$ and 3 prepare the way for this structure theorem.

2. The coextension. Let $S$ be any pseudo-inverse semigroup. In this section we shall show that there exist a pseudo-inverse semigroup $\mathcal{S}$ which has injective structure mappings and a homomorphism $\phi$ of $\delta$ onto $S$ such that the $\phi \phi^{-1}$-classes which contain idempotents are completely simple subsemigroups of $\mathcal{\delta}$. We shall factorize $\phi$ as a composition $\phi_{1} \phi_{2}$ of epimorphisms $\phi_{1}: \mathcal{S} \rightarrow \mathbf{S}$ and $\phi_{2}: \mathbf{S} \rightarrow S$ such that (i) $\phi_{1} \phi_{1}^{-1}$ is an idempotent-separating congruence on $\mathcal{S}$, and (ii) the $\phi_{2} \phi_{2}^{-1}$-classes which contain idempotents are rectangular bands.

We need the following result from [24].

THEOREM 2.1. Let $S$ be any pseudo-inverse semigroup, and let $\left(E, \omega^{l}, \omega^{r}\right)$ be the pseudo-semilattice which is determined by $S$. Let $\mathbf{S}=\{(e, x, f) \in E \times S \times E \mid x \in$ $e S \cap S f\}$, and define a multiplication on $\mathbf{S}$ by

$$
(e, x, f)(g, y, h)=(e, x y, h) .
$$

Then $\mathbf{S}$ is a pseudo-inverse semigroup, and the mapping $\phi_{2}: \mathbf{S} \rightarrow S,(e, x, f) \rightarrow x$ is a homomorphism of $\mathbf{S}$ onto $S$, such that the $\phi_{2} \phi_{2}^{-1}$ classes which contain idempotents are rectangular bands. Furthermore $\mathbf{E}=E(\mathbf{S})=\left\{(e, g, f) \in E \times E \times E \mid g \in \omega^{r}(e) \cap\right.$ $\left.\omega^{l}(f)\right\}$. In the pseudo-semilattice $\left(\mathbf{E}, \omega^{l}, \omega^{r}\right)$ which is determined by $\mathbf{S}$ we have

$$
(e, m, f) \omega^{r}(g, n, h)
$$

if and only if $e=g$ and $m \omega^{r} n$ in $S$, and

$$
(e, m, f) \omega^{l}(g, n, h)
$$

if and only if $f=h$ and $m \omega^{\prime} n$ in $S$. For any $e, f \in E, \mathbf{E}_{e, f}=\left\{(e, g, f) \mid g \in \omega^{r}(e) \cap\right.$ $\left.\omega^{l}(f)\right\}$ is a maximal subsemilattice of $\left(\mathbf{E}, \omega^{l}, \omega^{r}\right)$, and $\mathbf{E}_{e, f}$ has an identity element $(e, e \wedge f, f)$. The pseudo-semilattice $\left(\mathbf{E}, \omega^{l}, \omega^{r}\right)$ is the disjoint union of its maximal subsemilattices $\mathbf{E}_{e, f}, e, f \in E$.

COROLlaRY 2.2. The pseudo-semilattice $\left(\mathbf{E}, \omega^{l}, \omega^{r}\right)$ which is determined by $\mathbf{S}$ is of the form $\mathbf{E}=\left(L_{\lambda}, R_{i} ; M_{i \lambda} ; \phi_{i \lambda}, \psi_{i \lambda} ; I, \Lambda\right)$, where

(i) $I=\Lambda$,

(ii) $R_{i}=L_{i}=M_{i i}$ for all $i \in I=\Lambda$,

(iii) $\phi_{i i}=\psi_{i i}$ is the identity transformation on $M_{i i}$ for all $i \in I=\Lambda$,

(iv) for every $(i, j) \in I \times I, M_{i j}$ is a semilattice with an identity. 
Proof. Let us put $I=\Lambda=E$, and for all $(e, f) \in E \times E$, we put $M_{e, f}=\mathbf{E}_{e, f}$, $R_{e}=L_{e}=\mathbf{E}_{e, e}$, and

$$
\begin{array}{ll}
\psi_{e, f}: \mathbf{E}_{e, f} \rightarrow \mathbf{E}_{e, e}, & (e, g, f) \rightarrow(e, g \wedge e, e), \\
\phi_{e, f}: \mathbf{E}_{e, f} \rightarrow \mathbf{E}_{f, f}, & (e, g, f) \rightarrow(f, f \wedge g, f) .
\end{array}
$$

Then $\mathbf{E}=\left(L_{\lambda}, R_{i} ; M_{i \lambda} ; \phi_{i \lambda}, \psi_{i \lambda} ; I, \Lambda\right)$ is the pseudo-semilattice which is determined by $\mathbf{S}$, and the conditions (2.1) are satisfied.

Let us now suppose that $D$ is a pseudo-inverse semigroup such that the pseudosemilattice $\left(E(D), \omega^{l}, \omega^{r}\right)$ which is determined by $D$ is of the form

$$
E(D)=\left(L_{\lambda}, R_{i} ; M_{i \lambda} ; \phi_{i \lambda}, \psi_{i \lambda} ; I, \Lambda\right)
$$

where the conditions (2.1) are satisfied. For any $i, j \in I$ let $D_{i j}$ be the set of elements of $D$ which are $\Re$-related to some element of $M_{i i}$ and $\mathcal{L}$-related to some element of $M_{j j}$.

LEMma 2.3. $D$ is a rectangular band $I \times I$ of the semigroups $D_{i j},(i, j) \in I \times I$. For every $(i, j) \in I \times I, M_{i j}$ is the semilattice of idempotents of $D_{i j}$, and $D_{i j}$ is an order-ideal of $D, \leqslant$. For every $i \in I, D_{i i}$ is an inverse semigroup.

Proof. Immediate from Result 3.

If $x_{i j} \in D_{i j}$ is any element of $D$, then there exist idempotents $e_{i i} \in M_{i i}$ and $f_{j j} \in M_{j j}$ such that $e_{i i} \Re x_{i j} \ell f_{j j}$. The inverse of $x_{i j}$ which belongs to $R_{f_{j j}} \cap L_{e_{i i}}$ will be denoted by $x_{i j}^{-1}$. Observe that $x_{i j}^{-1} \in D_{j i}$, and, due to the fact that $M_{i i}$ and $M_{j j}$ are semilattices, $x_{i j}^{-1}$ is the unique inverse of $x_{i j}$ in $D_{j i}$.

LEMmA 2.4. For any $(i, j) \in I \times I$ the mapping $D_{i j} \rightarrow D_{j i}, x_{i j} \rightarrow x_{i j}^{-1}$ is an orderpreserving one-to-one mapping of $D_{i j}$ onto $D_{j i}$.

LEMMA 2.5. Let $x_{i j} \in D_{i j}$ be an element of $D$, and $\left\langle x_{i j}\right\rangle=\left\{y_{i j} \in D \mid y_{i j} \leqslant x_{i j}\right\}$. The mappings

$$
\begin{array}{ll}
\kappa_{1}:\left\langle x_{i j}\right\rangle \rightarrow\left\langle x_{i j} x_{i j}^{-1}\right\rangle, & y_{i j} \rightarrow y_{i j} y_{i j}^{-1}, \\
\kappa_{2}:\left\langle x_{i j}\right\rangle \rightarrow\left\langle x_{i j}^{-1} x_{i j}\right\rangle, & y_{i j} \rightarrow y_{i j}^{-1} y_{i j},
\end{array}
$$

are order-preserving one-to-one mappings onto the principal ideals $\left\langle x_{i j} x_{i j}^{-1}\right\rangle$ and $\left\langle x_{i j}^{-1} x_{i j}\right\rangle$ of $M_{i i}$ and $M_{j j}$ respectively.

Let $X$ be the set of order-ideals $Y_{i j}$ of $D_{i j}, i, j \in I$, where every $Y_{i j}$ is maximal for the property that the mappings $Y_{i j} \rightarrow M_{i i}, y_{i j} \rightarrow y_{i j} y_{i j}^{-1}$, and $Y_{i j} \rightarrow M_{j j}, y_{i j} \rightarrow y_{i j}^{-1} y_{i j}$, are injective order-preserving mappings onto ideals of $M_{i i}$ and $M_{j j}$ respectively. If $x_{i j} \in D_{i j}$ is any element of $D$, then it follows from Lemma 2.5 that there exists a $Y_{i j} \in X$ such that $x_{i j} \in Y_{i j}$. Thus $X \neq \square$, and $\cup_{Y_{i j} \in X} Y_{i j}=D$.

For any $Y_{i j} \in X$, let us denote

$$
Y_{i j}^{-1}=\left\{y_{i j}^{-1} \mid y_{i j} \in Y_{i j}\right\} \text {. }
$$

From Lemmas 2.4 and 2.5 we have $Y_{i j}^{-1} \subseteq D_{j i}$, and $Y_{i j} \in X$ if and only if $Y_{i j}^{-1} \in X$. It should be clear that, for all $i \in I, M_{i i}=M_{i i}^{-1} \in X$, since in this case we have, from Lemma 2.3, that $D_{i i}$ is an inverse semigroup. 
Let us consider a set $Z=\left\{I_{i j} \mid i, j \in I\right\}$, where $M_{i i}=I_{i i}$ for all $i \in I$ and $X \cap Z=\left\{I_{i i} \mid i \in I\right\}=\left\{M_{i i} \mid i \in I\right\}$. Let $\mathfrak{N}$ be the semigroup which is freely generated by the elements of $X \cup Z$, subject to the defining relations

$$
\begin{aligned}
& \text { (i) } I_{i j} I_{i k}=I_{i k}, \\
& \text { (ii) } I_{i j} I_{k j}=I_{i j}, \\
& \text { (iii) } I_{i j} Y_{i k}=Y_{i k}, \\
& \text { (iv) } Y_{i j} I_{k j}=Y_{i j}, \\
& \text { (v) } Y_{i j} Y_{i j}^{-1}=I_{i i} \text { for all } i, j, k \in I .
\end{aligned}
$$

LEMMA 2.6. $\mathfrak{T}$ is a completely simple semigroup and $Z=E(\mathfrak{T})$ is the set of idempotents of $\mathfrak{N}$.

For any $A \in X \cup Z$, let $\bar{A}=A$ if $A \in X$ and $\bar{A}=M_{i j}$ if $A=I_{i j} \in Z$. For any $X_{1}, \ldots, X_{n} \in X \cup Z$, let $\bar{X}_{1} \circ \cdots \circ \bar{X}_{n}$ denote the set of elements $x_{1} \cdots x_{n}$, where $x_{k} \in \bar{X}_{k}$ for all $k$. Thus $\circ$ is a complex multiplication [20, Chapter III] of subsets of D.

LEMMA 2.7. Let $X_{1}, \ldots, X_{n}$ be any elements of $X \cup Z$. Then no two different elements of $\bar{X}_{1} \circ \bar{X}_{2} \circ \ldots \circ \bar{X}_{n}$ are $\Re$ related in $D$.

Proof. Let us suppose that $x_{i_{\nu_{1}}} \cdots x_{i_{n} j_{n}}$ and $y_{i_{\nu_{1}}} \cdots y_{i_{n} j_{n}}$ are different $\Re$-related elements of $\bar{X}_{1} \circ \bar{X}_{2} \circ \cdots \circ \bar{X}_{n}$, where for all $k, x_{i_{k} j_{k}}, y_{i_{k} j_{k}} \in Y_{i_{k} j_{k}}$ if $X_{k}=Y_{i_{k} j_{k}} \in X$, and $x_{i_{k} j_{k}}, y_{i_{k} j_{k}} \in M_{i_{k} j_{k}}$ if $X_{k}=I_{i_{k} j_{k}} \in Z$. Let us put

$$
h=\left(x_{i_{2} j_{2}} \cdots x_{i_{n} j_{n}}\right)\left(x_{i_{2} j_{2}} \cdots x_{i_{n} j_{n}}\right)^{-1} \wedge x_{i_{\nu_{1}}}^{-1} x_{i_{\nu_{1}} j_{1}}
$$

and

$$
g=\left(y_{i_{2} j_{2}} \cdots y_{i_{n} j_{n}}\right)\left(y_{i_{2} j_{2}} \cdots y_{i_{n} j_{n}}\right)^{-1} \wedge y_{i_{\nu_{1}}}^{-1} y_{i_{\nu_{1}}} .
$$

Then $g, h \in M_{i_{2} j_{1}}, y_{i_{\nu_{1}}} g \in D_{i_{\nu_{1}}}, x_{i_{\nu_{1}}} h \in D_{i_{\nu_{1}}}$, and

$$
x_{i_{\nu_{1}}} h \Re x_{i_{j_{1}}} \cdots x_{i_{n} j_{n}} \Re y_{i_{i_{1}}} \cdots y_{i_{n} j_{n}} \Re y_{i_{\nu_{1}}} g \text {. }
$$

Since $h \omega^{l} x_{i_{\nu_{1}}}^{-1} x_{i_{\nu_{1}}}$, we must have $x_{i_{\nu_{1}}} h \leqslant x_{i_{\nu_{1}}}$, and since $g \omega^{l} y_{i_{\nu_{1}}}^{-1} y_{i_{\nu_{1}}}$, we must have $y_{i_{1},} g \leqslant y_{i_{\nu_{1}}}$. Thus, $x_{i_{j_{1}}} h, y_{i_{\nu_{1}}} g \in Y_{i_{\nu_{1}}}$ if $X_{1}=Y_{i_{j_{1}}} \in X$, and $x_{i_{j_{1}}} h, y_{i_{\nu_{1}}} g \in M_{i_{\nu_{1}}}$ if $X_{1}=I_{i_{\nu_{1}}} \in Z$. Since no two different elements of $Y_{i_{\nu_{1}}}$ or $\bar{I}_{i_{j_{1}}}$ can be $\mathcal{R}$-related in $D$,

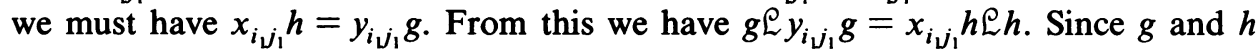
belong to the semilattice $M_{i_{2} j_{1}}$, we conclude $g=h$ and so

$$
h\left(x_{i_{2} j_{2}} \cdots x_{i_{n} j_{n}}\right) \Re h=g \Re g\left(y_{i_{2} j_{2}} \cdots y_{i_{n} j_{n}}\right) \text {. }
$$

The inner left translation with $x_{i_{\nu_{1}}} h=y_{i_{j_{1}}} g$ maps the $R$-class of $h=g$ bijectively onto the $\Re$-class of $x_{i_{j_{1}}} h=y_{i_{\nu_{1}}} g$ by Green's lemma (Lemma 2.2 of [7]). Since

$$
\begin{aligned}
\left(x_{i_{\nu_{1}}} h\right)\left(h x_{i_{2} j_{2}} \cdots x_{i_{n} j_{n}}\right) & =x_{i_{\nu_{1}} j_{1}} x_{i_{2} j_{2}} \cdots x_{i_{n} j_{n}} \\
& \neq y_{i_{\nu_{1}} y_{1}} y_{i_{2} j_{2}} \cdots y_{i_{n} j_{n}}=\left(y_{i_{\nu} j_{1}} g\right)\left(g y_{i_{2} j_{2}} \cdots y_{i_{n} j_{n}}\right)
\end{aligned}
$$


we have that $h x_{i_{2} j_{2}} \cdots x_{i_{n} j_{n}}$ and $g y_{i_{2} j_{2}} \cdots y_{i_{n} j_{n}}$ must be two different $\Re$-related elements of $D$. Since $h \omega^{r} x_{i_{2} j_{2}} x_{i_{2} j_{2}}^{-1}$ and $g \omega^{r} y_{i_{2} j_{2}} y_{i_{2} j_{2}}^{-1}$, we have $h x_{i_{2} j_{2}} \leqslant x_{i_{2} j_{2}}$ and $g y_{i_{2} j_{2}} \leqslant$ $y_{i_{2} j_{2}}$. Therefore $h x_{i_{i_{2} j_{2}}}, g y_{i_{2} j_{2}} \in Y_{i_{2} j_{2}}$ if $X_{2}=Y_{i_{2} j_{2}} \in X$ and $h x_{i_{2} j_{2}}, g y_{i_{2} j_{2}} \in M_{i_{2} j_{2}}$ if $X_{2}=$ $I_{i_{2} j_{2}} \in Z$. Hence $\left(h x_{i_{2} j_{2}}\right) \cdots x_{i_{n} j_{n}}$ and $\left(g y_{i_{2} j_{2}}\right) \cdots y_{i_{n} j_{n}}$ are different $\Re$-related elements of $\bar{X}_{2} \circ \ldots \circ \bar{X}_{n}$. By induction we can then show that $Y_{i_{n} j_{n}}$ or $M_{i_{n} j_{n}}$ contains two different $\Re$-related elements. This is clearly impossible, and our supposition is false. Therefore the lemma holds.

Let $\mathscr{F}_{X \cup Z}$ be the free semigroup on the set $X \cup Z$, and let $\rho_{0}$ be the relation on $\mathscr{F}_{X \cup Z}$ which consists of the pairs $\left(I_{i j} I_{i k}, I_{i k}\right),\left(I_{i j} I_{k j}, I_{i j}\right),\left(I_{i j} Y_{i k}, Y_{i k}\right),\left(Y_{i j} I_{k j}, Y_{i j}\right)$, $\left(Y_{i j} Y_{i j}^{-1}, I_{i i}\right), i, j, k \in I$. If $\rho$ is the congruence relation on $\mathscr{F}_{X \cup Z}$ which is generated by $\rho_{0}$, then we can identify $\mathscr{F}_{X \cup Z} / \rho$ with the completely simple semigroup $\mathfrak{T}$. A word $W$ of $\mathscr{F}_{X \cup Z}$ will be called reduced if $W$ cannot be made shorter by an application of an elementary $\rho_{0}$-transition. An elementary $\rho_{0}$-transition will be called decreasing if it decreases the length of the word on which it acts.

LEMMA 2.8. Every $\rho$-class on $\mathscr{F}_{X \cup Z}$ contains a unique reduced word. If $X_{1} \cdots X_{n}$ is any word of $\mathscr{F}_{X \cup Z}$, and if $V_{1} \cdots V_{k}$ is the reduced word in the $\rho$-class of $X_{1} \cdots X_{n}$, then $\bar{X}_{1} \circ \bar{X}_{2} \circ \cdots \circ \bar{X}_{n} \subseteq \bar{V}_{1} \circ \cdots \circ \bar{V}_{k}$.

SKETCH OF THE PROOF. Every word of $\mathscr{F}_{X \cup Z}$ can be transformed into a reduced word by subsequent applications of elementary $\rho_{0}$-transitions, and so every $\rho$-class contains at least one reduced word.

Let $W$ be any word of $\mathscr{F}_{X \cup Z}$. Let us suppose that we can transform $W$ into the reduced word $W_{1}$ by an application of the decreasing elementary $\rho_{0}$-transitions $\alpha_{1}, \ldots, \alpha_{s}$, and into the reduced word $W_{2}$ by an application of the decreasing elementary $\rho_{0}$-transitions $\beta_{1}, \ldots, \beta_{t}$. We show by induction on the length of $W$ that $W_{1}=W_{2}$. It is easy to see that this statement is true if $W$ is a word of length 1 or 2 . Let the statement be true for any word which has a length which is smaller than the length of $W$, and let $W$ be a word of length $n$, with $n \geqslant 3$. If $\alpha_{1}: W \rightarrow W^{\prime}, \beta_{1}$ : $W \rightarrow W^{\prime \prime}$, then $W^{\prime}$ and $W^{\prime \prime}$ are both words of length $n-1$. One can show that either $W^{\prime}=W^{\prime \prime}$ or that $W^{\prime}$ and $W^{\prime \prime}$ can both be transformed into the same shorter word by an application of decreasing elementary $\rho_{0}$-transitions. This allows us to apply the induction hypothesis, and to conclude that $W_{1}=W_{2}$.

Let us now suppose that $T_{1}$ and $T_{2}$ are $\rho$-related reduced words. Then $T_{1}$ can be transformed into $T_{2}$ by subsequent appligations of elementary $\rho_{0}$-transitions,

$$
T_{1}=W_{1} \rightarrow W_{2} \cdots W_{k} \rightarrow W_{k+1} \cdots W_{u} \rightarrow W_{u+1}=T_{2} .
$$

For any $k \in\{1, \ldots, u+1\}$, let $W_{k}^{\prime}$ be a reduced word which is obtained from $W_{k}$ by subsequent applications of decreasing elementary $\rho_{0}$-transitions. Using the observation mentioned above, we have $W_{1}^{\prime}=W_{2}^{\prime}=\cdots=W_{u+1}^{\prime}$. Obviously $T_{1}=W_{1}^{\prime}$ and $T_{2}=W_{u+1}^{\prime}$, and so $T_{1}=T_{2}$ is the unique reduced word of its $\rho$-class.

Let $X_{1} \cdots X_{n}$ be any word of $\mathscr{F}_{X \cup Z}$, and let $V_{1} \cdots V_{k}$ be the reduced word in the $\rho$-class of $X_{1} \cdots X_{n}$. In order to show that $\bar{X}_{1} \circ \cdots \circ \bar{X}_{n} \subseteq \bar{V}_{1} \circ \cdots \circ \bar{V}_{k}$ it suffices to show that (i) $M_{i j} \circ M_{i k} \subseteq M_{i k}$, (ii) $M_{i j} \circ M_{k j} \subseteq M_{i j}$, (iii) $M_{i j} \circ Y_{i k} \subseteq Y_{i k}$, (iv) $Y_{i j} \circ M_{k j} \subseteq Y_{i j}$, (v) $Y_{i j} \circ Y_{i j}^{-1} \subseteq M_{i i}$, for all $i, j, k \in I$. Case (i) is similar to case (iii), case (ii) is similar to case (iv), cases (iii) and (iv) can be treated in a dual way. Therefore we shall concentrate on cases (iii) and (v). 
Let $e_{i j} \in M_{i j}$ and $y_{i k} \in Y_{i k}$. Then $e_{i j} y_{i k}=e_{i j}\left(y_{i k} y_{i k}^{-1} \wedge e_{i j}\right) y_{i k}$. Since $y_{i k} y_{i k}^{-1} \wedge$ $e_{i j} \omega^{l} e_{i j}$, and $y_{i k} y_{i k}^{-1} \wedge e_{i j} \in M_{i j}$, we have $y_{i k} y_{i k}^{-1} \wedge e_{i j} \omega e_{i j}$, and so

$$
e_{i j} y_{i k}=\left(y_{i k} y_{i k}^{-1} \wedge e_{i j}\right) y_{i k}=\left(y_{i k} y_{i k}^{-1} \wedge e_{i j}\right)\left(y_{i k} y_{i k}^{-1}\right) y_{i k} \text {. }
$$

Since $\left(y_{i k} y_{i k}^{-1} \wedge e_{i j}\right)\left(y_{i k} y_{i k}^{-1}\right) \omega y_{i k} y_{i k}^{-1}$, we have $e_{i j} y_{i k} \leqslant y_{i k}$, and so $e_{i j} y_{i k} \in Y_{i k}$. We conclude that $M_{i j} \circ Y_{i k} \subseteq Y_{i k}$.

Let $y_{i j} \in Y_{i j}$ and $v_{i j}^{-1} \in Y_{i j}^{-1}$, where $v_{i j} \in Y_{i j}$. Then

$$
y_{i j} v_{i j}^{-1}=y_{i j}\left(v_{i j}^{-1} v_{i j} \wedge y_{i j}^{-1} y_{i j}\right) v_{i j}^{-1} .
$$

We have $y_{i j}\left(v_{i j}^{-1} v_{i j} \wedge y_{i j}^{-1} y_{i j}\right) \mathcal{L}\left(v_{i j}^{-1} v_{i j} \wedge y_{i j}^{-1} y_{i j}\right) \Re\left(v_{i j}^{-1} v_{i j} \wedge y_{i j}^{-1} y_{i j}\right) v_{i j}^{-1}$,

$$
y_{i j}\left(v_{i j}^{-1} v_{i j} \wedge y_{i j}^{-1} y_{i j}\right) \leqslant y_{i j}, \quad\left(v_{i j}^{-1} v_{i j} \wedge y_{i j}^{-1} y_{i j}\right) v_{i j}^{-1} \leqslant v_{i j}^{-1},
$$

and so $y_{i j}\left(v_{i j}^{-1} v_{i j} \wedge y_{i j}^{-1} y_{i j}\right) \in Y_{i j},\left(v_{i j}^{-1} v_{i j} \wedge y_{i j}^{-1} y_{i j}\right) v_{i j}^{-1} \in Y_{i j}^{-1}$, and

$$
\left(v_{i j}^{-1} v_{i j} \wedge y_{i j}^{-1} y_{i j}\right) v_{i j}^{-1}=\left(y_{i j}\left(v_{i j}^{-1} v_{i j} \wedge y_{i j}^{-1} y_{i j}\right)\right)^{-1} \text {. }
$$

Thus

$$
y_{i j} v_{i j}^{-1}=\left(y_{i j}\left(v_{i j}^{-1} v_{i j} \wedge y_{i j}^{-1} y_{i j}\right)\right)\left(y_{i j}\left(v_{i j}^{-1} v_{i j} \wedge y_{i j}^{-1} y_{i j}\right)\right)^{-1} \in M_{i i} .
$$

We conclude that $Y_{i j} \circ Y_{i j}^{-1} \subseteq M_{i i}$.

This completes the proof of the lemma.

Let us now consider the set $\mathscr{D}$ consisting of the elements $\left(e_{i i}, X_{1} \cdots X_{n}\right) \in$ $\left(\cup_{i \in I} M_{i i}\right) \times \mathfrak{K}$ for which the following condition is satisfied:

if $V_{1} \cdots V_{k}$ is the unique reduced word in the $\rho$-class of the word $X_{1} \cdots X_{n}$ of $\mathscr{F}_{X \cup Z}$, then $R_{e_{i i}} \cap \bar{V}_{1} \circ \cdots \circ \bar{V}_{k} \neq \square$.

Let $\left(e_{i i}, X_{1} \cdots X_{n}\right)$ be any element of $\mathscr{Q}$, and let $V_{1} \cdots V_{k}$ be the reduced word in the $\rho$-class of $X_{1} \cdots X_{n}$. By Lemmas 2.7 and 2.8 there exists a unique element $x \in D$ such that $R_{e_{i i}} \cap \bar{V}_{1} \circ \cdots \circ \bar{V}_{k}=\{x\}$, and so the mapping $\psi: \mathscr{D} \rightarrow D,\left(e_{i i}, X_{1}\right.$ $\left.\cdots X_{n}\right) \rightarrow x$ is well defined.

On $\mathscr{D}$ we define a multiplication in the following way. If $P=\left(e_{i i}, X_{1} \cdots X_{n}\right)$ and $Q=\left(f_{j j}, Y_{1} \cdots Y_{m}\right)$ are any elements of $\left.\mathscr{Q}\right)$, then

$$
P Q=\left((P \psi)(Q \psi)((P \psi)(Q \psi))^{-1}, X_{1} \cdots X_{n} Y_{1} \cdots Y_{m}\right)
$$

LEMMA 2.9. $\mathscr{D}$ is a semigroup and $\psi$ is a homomorphism of $\mathscr{D}$ onto $D$.

Proof. Let $P=\left(e_{i i}, X_{1} \cdots X_{n}\right)$ and $Q=\left(f_{j j}, Y_{1} \cdots Y_{m}\right)$ be any elements of $\mathscr{Q}$. Let $P \psi=x, Q \psi=y$, and let $U_{1} \cdots U_{l}$ be the unique reduced word in the $\rho$-class of the word $X_{1} \cdots X_{n} Y_{1} \cdots Y_{m}$ of $\mathscr{F}_{X \cup Z}$. By Lemma 2.8

$$
(P \psi)(Q \psi)=x y \in \bar{U}_{1} \circ \cdots \circ \bar{U}_{l},
$$

and so $x y=R_{((P \psi)(Q \psi))((P \psi)(Q \psi))^{-1}} \cap \bar{U}_{1} \circ \ldots \circ \bar{U}_{l}$; therefore

$$
\left.\left((P \psi)(Q \psi)((P \psi)(Q \psi))^{-1}, X_{1} \cdots X_{n} Y_{1} \cdots Y_{m}\right) \in \mathscr{D}\right)
$$

and the multiplication given by (2.4) is well defined. Moreover $(P Q) \psi=x y=$ $(P \psi)(Q \psi)$; hence $\psi$ is a homomorphism. If $x_{i j} \in D_{i j}$ is any element of $D$, then it follows from Lemma 2.5 that there exists a $Y_{i j} \in X$ such that $x_{i j} \in Y_{i j}$. Then $\left(x_{i j} x_{i j}^{-1}, Y_{i j}\right) \in \mathscr{D}$ and $\left(x_{i j} x_{i j}^{-1}, Y_{i j}\right) \psi=x_{i j}$. Thus $\psi$ is surjective. 
If $T=\left(g_{h h}, Z_{1} \cdots Z_{k}\right)$ is any other element of $\mathscr{D}$, then it follows from $((P Q) T) \psi$ $=(P \psi)(Q \psi)(T \psi)=(P(Q T)) \psi$ that

$$
\begin{aligned}
(P Q) T & =\left(((P \psi)(Q \psi)(T \psi))((P \psi)(Q \psi)(T \psi))^{-1}, X_{1} \cdots X_{n} Y_{1} \cdots Y_{m} Z_{1} \cdots Z_{k}\right) \\
& =P(Q T) .
\end{aligned}
$$

Thus the multiplication given by (2.4) is associative.

LEMMA 2.10. $D$ is a regular semigroup and

$$
E(\mathscr{D})=\left\{\left(e_{i i}, I_{i j}\right) \mid i, j \in I, e_{i i} \in M_{i i}, R_{e_{i i}} \cap M_{i j} \neq \square\right\} .
$$

Proof. Since $E(\mathscr{N})=Z$ by Lemma 2.6 , we immediately have that $E(\mathscr{D})$ is given by (2.5).

Let $P=\left(e_{i i}, X_{1} \cdots X_{n}\right)$ be any element of $\mathscr{Q}$. We can always suppose that $X_{1} \cdots X_{n}$ is a reduced word of $\mathscr{F}_{X \cup Z}$. Then $P \psi=x_{i_{1} j_{1}} \cdots x_{i_{n} j_{n}} \in \bar{X}_{1} \circ \cdots \circ \bar{X}_{n}$ for some $x_{i_{k} j_{k}} \in \bar{X}_{k}, k=1, \ldots, n$, where for all $k, X_{k}=Y_{i_{k} j_{k}}$ or $I_{i_{k} j_{k}}$. Let

$$
f_{j j}=(P \psi)^{-1}(P \psi)=\left(x_{i_{\nu} j_{1}} \cdots x_{i_{n} j_{n}}\right)^{-1}\left(x_{i_{j_{1}}} \cdots x_{i_{n} j_{n}}\right) \text {. }
$$

Clearly $i=i_{1}$ and $j=j_{n}$ by Lemma 2.3. Let us put $X_{k}^{-1}=Y_{i_{k} j_{k}}^{-1}$ if $X_{k}=Y_{i_{k} j_{k}}$ and $X_{k}^{-1}=I_{j_{k} j_{k}} I_{i_{k} i_{k}}$ if $X_{k}=I_{i_{k} j_{k}} ; X_{k}^{-1}$ is the inverse in $\mathfrak{N}$ of $X_{k}$ which belongs to the maximal subgroup which has identity $I_{j_{k} i_{k}}$. We shall show that

$$
Q=\left(f_{j_{n} j_{n}}, X_{n}^{-1} I_{i_{n} j_{n-1}} X_{n-1}^{-1} \cdots X_{2}^{-1} I_{i_{2} j_{1}} X_{1}^{-1}\right)
$$

belongs to $\mathscr{D}$ and is an inverse of $P$ where $P Q=\left(e_{i_{1} i_{1}}, I_{i_{1} i_{1}}\right)$ and $Q P=\left(f_{j_{n} j_{n}}, I_{j_{n} j_{n}}\right)$. We show this by induction on $n$.

Let us first consider the case $n=1$ and $P=\left(e_{i i}, I_{i j}\right)$. Then $P \psi=g_{i j}=e_{i j} \psi_{i j}^{-1}$ is

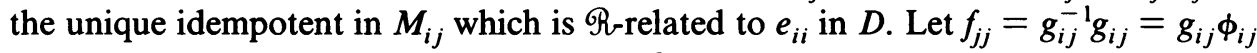

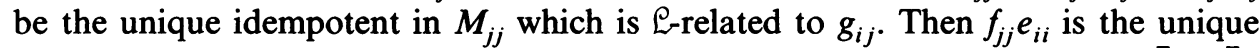

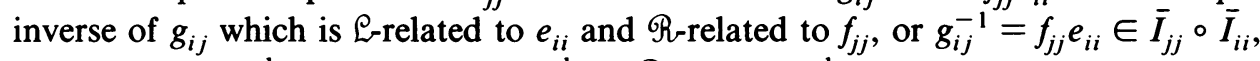
where $I_{j j} I_{i i}=I_{i j}^{-1}$. Thus $Q=\left(f_{j j}, I_{i j}^{-1}\right) \in \mathscr{D}, Q \psi=g_{i j}^{-1}$,

$$
\begin{gathered}
P Q=\left((P \psi)(Q \psi)((P \psi)(Q \psi))^{-1}, I_{i j} I_{i j}^{-1}\right)=\left(g_{i j} g_{i j}^{-1}, I_{i i}\right)=\left(e_{i i}, I_{i i}\right), \\
Q P=\left((Q \psi)(P \psi)((Q \psi)(P \psi))^{-1}, I_{i j}^{-1} I_{i j}\right)=\left(g_{i j}^{-1} g_{i j}, I_{j j}\right)=\left(f_{j j}, I_{j j}\right), \\
P Q P=P, \quad Q P Q=Q .
\end{gathered}
$$

Let us next consider the case $n=1$ and $P=\left(e_{i i}, Y_{i j}\right)$. Then $P \psi=y_{i j}$ is the unique element in the $\Re$-class of $e_{i i}$ which belongs to $Y_{i j}$. Let $f_{j j}=y_{i j}^{-1} y_{i j}$. Clearly $y_{i j}^{-1} \in Y_{i j}$, $Q=\left(f_{j j}, Y_{i j}^{-1}\right) \in \mathscr{D}$, and $Q \psi=y_{i j}^{-1}$. It is again easy to see that $P Q=\left(e_{i i}, I_{i i}\right)$, $Q P=\left(f_{j j}, I_{j j}\right), P Q P=P$ and $Q P Q=Q$. We conclude that our assumption holds if $n=1$.

Let us now suppose that $n>1$, and that our assumption holds for all $l, l<n$. Let us again consider $P=\left(e_{i_{1}, i_{1}}, X_{1} \cdots X_{n}\right)$ where $X_{1} \cdots X_{n}$ is reduced, and let $P \psi=$ $x_{i_{\nu_{1}}} \cdots x_{i_{n} j_{n}} \in \bar{X}_{1} \circ \cdots \circ \bar{X}_{n}$, where $x_{i_{k} j_{k}} \in \bar{X}_{k}, k=1, \ldots, n$. Let $T=\left(g_{i_{2} i_{2}}, X_{2}\right.$ $\left.\cdots X_{n}\right)$ where $g_{i_{2} i_{2}}=\left(x_{i_{2} j_{2}} \cdots x_{i_{n} j_{n}}\right)\left(x_{i_{2} j_{2}} \cdots x_{i_{n} j_{n}}\right)^{-1}$ and let

$$
U=\left(h_{j_{n} j_{n}}, X_{n}^{-1} I_{i_{n} j_{n-1}} X_{n-1}^{-1} \cdots X_{3}^{-1} I_{i_{3} j_{2}} X_{2}^{-1}\right)
$$


where $h_{j_{n} j_{n}}=\left(x_{i_{2} j_{2}} \cdots x_{i_{n} j_{n}}\right)^{-1}\left(x_{i_{2} j_{2}} \cdots x_{i_{n} j_{n}}\right)$. By the induction hypothesis we have that $T$ and $U$ are mutually inverse in $\mathscr{Q})$ and that $T U=\left(g_{i_{2} i_{2}}, I_{i_{2} i_{2}}\right), U T=\left(h_{j_{n} j_{n}}, I_{j_{n} j_{n}}\right)$. Clearly

$$
\begin{gathered}
(T U) \psi=g_{i_{2} i_{2}}, \quad(U T) \psi=h_{j_{n} j_{n}}, \\
T \psi=x_{i_{2} j_{2}} \cdots x_{i_{n} j_{n}}, \quad U \psi=\left(x_{i_{2} j_{2}} \cdots x_{i_{n} j_{n}}\right)^{-1} .
\end{gathered}
$$

Let

$$
r_{i_{2} j_{1}}=\left(x_{i_{2} j_{2}} \cdots x_{i_{n} j_{n}}\right)\left(x_{i_{2} j_{2}} \cdots x_{i_{n} j_{n}}\right)^{-1} \wedge x_{i_{\nu_{1}}}^{-1} x_{i_{j_{1}}}
$$

and let us consider $\left(x_{i_{2} j_{2}} \cdots x_{i_{n} j_{n}}\right)^{-1} r_{i_{2} j_{1}} x_{i_{\nu_{1}}}^{-1}$. We know that $\left(x_{i_{2} j_{2}} \cdots x_{i_{n} j_{n}}\right)^{-1} r_{i_{2} j_{1}} x_{i_{j_{1}} j_{1}}^{-1}$ is an inverse of $x_{i_{j_{1}}}\left(x_{i_{2} j_{2}} \cdots x_{i_{n} j_{n}}\right)$ from [26], and since, by Lemma 2.3, $\left(x_{i_{2} j_{2}} \cdots x_{i_{n} j_{n}}\right)^{-1} r_{i_{2} j_{1}} x_{i_{\nu_{1}}}^{-1} \in D_{j_{n} i_{1}}$, it follows that

$$
\left(x_{i_{2} j_{2}} \cdots x_{i_{n} j_{n}}\right)^{-1} r_{i_{2} j_{1}} x_{i_{j_{1}}}^{-1}=\left(x_{i_{\nu_{1}}} \cdots x_{i_{n} j_{n}}\right)^{-1} \text {. }
$$

Obviously $\left(\left(r_{i_{2} j_{1}} x_{i_{1} j_{1}}^{-1}\right)\left(r_{i_{2} j_{1}} x_{i_{j_{1}}}^{-1}\right)^{-1}, I_{i_{2} j_{1}} X_{1}^{-1}\right) \in \mathscr{D}$ and

$$
\left(\left(r_{i_{2} j_{1}} x_{i_{j_{1}}}^{-1}\right)\left(r_{i_{2} j_{1}} x_{i_{\nu_{1}}}^{-1}\right)^{-1}, I_{i_{2} j_{1}} X_{1}^{-1}\right) \psi=r_{i_{2} j_{1}} x_{i_{\nu_{1}}}^{-1} \text {. }
$$

Let us put $Q=U\left(\left(r_{i_{2} j_{1}} x_{i_{j_{1}}}^{-1}\right)\left(r_{i_{2} j_{1}} x_{i_{1} j_{1}}^{-1}\right)^{-1}, I_{i_{2} j_{1}} X_{1}^{-1}\right)$. From

$$
Q \psi=(U \psi)\left(r_{i_{2} j_{1}} x_{i_{j_{1}}}^{-1}\right)=\left(x_{i_{2} j_{2}} \cdots x_{i_{n} j_{n}}\right)^{-1} r_{i_{2} j_{1}} x_{i_{\nu_{1}} j_{1}}^{-1}=\left(x_{i_{j_{1}}} \cdots x_{i_{n} j_{n}}\right)^{-1}
$$

we have $(Q \psi) \Re\left(x_{i_{1} j_{1}} \cdots x_{i_{n} j_{n}}\right)^{-1}\left(x_{i_{1} j_{1}} \cdots x_{i_{n} j_{n}}\right)=f_{j_{n} j_{n}}$. Thus $Q=$ $\left(f_{j_{n} j_{n}}, X_{n}^{-1} I_{i_{n} j_{n-1}} X_{n-1}^{-1} \cdots X_{2}^{-1} I_{i_{2} j_{1}} X_{1}^{-1}\right)$ belongs to $\mathscr{D}$. Moreover, $(P Q) \psi=$ $\left(x_{i_{1} j_{1}} \cdots x_{i_{n} j_{n}}\right)\left(x_{i_{1} j_{1}} \cdots x_{i_{n} j_{n}}\right)^{-1}=e_{i_{1} i_{1}}$ and $(Q P) \psi=\left(x_{i_{j_{1}}} \cdots x_{i_{n} j_{n}}\right)^{-1}\left(x_{i_{j_{1}}} \cdots x_{i_{n} j_{n}}\right)$ $=f_{j_{n} j_{n}}$, whereas from $T U=\left(g_{i_{2} i_{2}}, I_{i_{2} i_{2}}\right)$ and $U T=\left(h_{j_{n} j_{n}}, I_{j_{n} j_{n}}\right)$ it follows that

$$
\begin{aligned}
& \left(X_{2} \cdots X_{n}\right)\left(X_{n}^{-1} I_{i_{n} j_{n-1}} X_{n-1}^{-1} \cdots X_{2}^{-1}\right)=I_{i_{2} i_{2}}, \\
& \left(X_{n}^{-1} I_{i_{n} j_{n-1}} X_{n-1}^{-1} \cdots X_{2}^{-1}\right)\left(X_{2} \cdots X_{n}\right)=I_{j_{n} j_{n}},
\end{aligned}
$$

and thus

$$
\begin{aligned}
& \left(X_{1} \cdots X_{n}\right)\left(X_{n}^{-1} I_{i_{n} j_{n-1}} X_{n-1}^{-1} \cdots X_{2}^{-1} I_{i_{2} j_{1}} X_{1}^{-1}\right) \\
& =X_{1} I_{i_{2} i_{2} I_{2} j_{1}} X_{1}^{-1}=X_{1} I_{i_{2} j_{1}} X_{1}^{-1}=X_{1} X_{1}^{-1}=I_{i_{1} i_{1}}, \\
& \left(X_{n}^{-1} I_{i_{n} j_{n-1}} X_{n-1}^{-1} \cdots X_{2}^{-1} I_{i_{2} j_{1}} X_{1}^{-1}\right)\left(X_{1} \cdots X_{n}\right) \\
& =\left(X_{n}^{-1} I_{i_{n} j_{n-1}} X_{n-1}^{-1} \cdots X_{2}^{-1}\right) I_{i_{2} j_{1} I_{j_{1}}}\left(X_{2} \cdots X_{n}\right) \\
& =\left(X_{n}^{-1} I_{i_{n} j_{n-1}} X_{n-1}^{-1} \cdots X_{2}^{-1}\right)\left(X_{2} \cdots X_{n}\right)=I_{j_{n} j_{n}} .
\end{aligned}
$$

We conclude that $P Q=\left(e_{i_{1} i_{1}}, I_{i_{1} i_{1}}\right), Q P=\left(f_{j_{n} j_{n}}, I_{j_{n} j_{n}}\right)$, and $P Q P=P, Q P Q=Q$.

This completes the proof of this lemma.

LEMMA 2.11. $\psi \psi^{-1}$ is an idempotent-separating congruence on 2 . 
Proof. Let $\left(e_{i i}, I_{i j}\right)$ and $\left(f_{m m}, I_{m n}\right)$ be any idempotents $\mathscr{D}$, and let us suppose that $\left(e_{i i}, I_{i j}\right) \psi=\left(f_{m m}, I_{m n}\right) \psi$. Since $\left(e_{i i}, I_{i j}\right) \psi \in D_{i j}$ and $\left(f_{m m}, I_{m n}\right) \psi \in D_{m n}$ we must have $i=m, j=n,\left(f_{m m}, I_{m n}\right)=\left(f_{i i}, I_{i j}\right)$ and

$$
\left(e_{i i}, I_{i j}\right) \psi=g_{i j}=\left(f_{i i}, I_{i j}\right) \psi \text {. }
$$

Furthermore $e_{i i}=g_{i j} \psi_{i j}=f_{i i}, g_{i j}$ is the unique element in $R_{e_{i i}} \cap \bar{I}_{i j}=R_{f_{i i}} \cap \bar{I}_{i j}$. We conclude that $\left(e_{i i}, I_{i j}\right)=\left(f_{m m}, I_{m n}\right)$. Hence $\psi \psi^{-1}$ is idempotent-separating.

LEMMA 2.12. D has injective structure mappings.

Proof. The proof immediately follows from Result 4.

LEMma 2.13. The mapping $\mathscr{D} \rightarrow \mathfrak{T},\left(e_{i i}, X_{1} \cdots X_{n}\right) \rightarrow X_{1} \cdots X_{n}$ induces the least primitive congruence on $\mathscr{D}$ and $\mathscr{T}$ is the greatest completely simple homomorphic image of $\mathscr{D}$.

Proof. Since $\mathfrak{K}$ is a completely simple semigroup, the above considered homomorphism induces a primitive congruence $\gamma$ on $\mathscr{D}$, and $\leqslant \circ(\leqslant)^{-1} \subseteq \gamma$.

If $P$ and $Q$ are any elements of $\mathscr{D}$ such that $Q \leqslant P$ in $\mathscr{D}$, then it follows from $Q \psi \leqslant P \psi$ in $D$ that $P=\left(e_{i i}, X_{1} \cdots X_{n}\right), Q=\left(f_{i i}, Y_{1} \cdots Y_{m}\right)$ where $f_{i i} \leqslant e_{i i}$ in $M_{i i}$, and $Q=\left(f_{i i}, I_{i i}\right) P$. We then conclude that $Y_{1} \cdots Y_{m}=I_{i i} X_{1} \cdots X_{n}=X_{1} \cdots X_{n}$. Thus $\leqslant \circ(\leqslant)^{-1}=\gamma$.

REMARK 2.14. Every element in the greatest completely simple homomorphic image of $\mathscr{D}$ which is a product of idempotents can be written in a unique way as a product $I_{i_{\nu_{1}}} \cdots I_{i_{n} j_{n}}$, where for each $1 \leqslant k \leqslant n-1, i_{k} \neq i_{k+1}$ and $j_{k} \neq j_{k+1}$. If $P$ is any element of $\mathscr{D}$ such that $P \gamma^{\#}=I_{i_{\nu_{1}}} \cdots I_{i_{n} j_{n}}$, where for each $1 \leqslant k \leqslant n-1$, $i_{k} \neq i_{k+1}$ and $j_{k} \neq j_{k+1}$, then $P$ is of the form $\left(e_{i_{1} i_{1}}, I_{i_{1} j_{1}} \cdots I_{i_{n} j_{n}}\right)$. Since $I_{i_{i_{1}}} \cdots I_{i_{n} j_{n}}$ is a reduced word of $\mathscr{F}_{X \cup Z}, P \psi$ is of the form $e_{i_{\nu} j_{1}} \cdots e_{i_{n} j_{n}}, e_{i_{k} j_{k}} \in M_{i_{k} j_{k}}$, where $e_{i_{\nu} j_{1}} \cdots e_{i_{n} j_{n}} \Re e_{i_{1} i_{1}}$ in $\mathscr{Q}$. Putting $e_{i_{k} j_{k}} \psi_{i_{k} j_{k}}=f_{i_{k} i_{k}}$ for every $1 \leqslant k \leqslant n$, we then have

$$
P=\left(f_{i_{1} i_{1}}, I_{i_{j_{1}}}\right) \cdots\left(f_{i_{n} i_{n}}, I_{i_{n} j_{n}}\right)
$$

where $\left(f_{i_{k} i_{k}}, I_{i_{k} j_{k}}\right) \gamma=I_{i_{k} j_{k}}$

We summarize Lemmas 2.9-2.12 in the following theorem.

THEOREM 2.15. Let $D$ be a pseudo-inverse semigroup, and suppose that the pseudosemilattice $\left(E(D), \omega^{l}, \omega^{r}\right)$ which is determined by $D$ satisfies the conditions $(2.1)$. Then there exist a pseudo-inverse semigroup $\mathscr{D}$ which has injective structure mappings and a homomorphism $\psi$ of $\mathscr{D}$ onto $D$ such that $\psi \psi^{-1}$ is an idempotent-separating congruence on $\mathscr{D}$.

Remark that if $E(D)$ reduces to a semilattice $(|I|=|\Lambda|=1)$ this theorem states that every inverse semigroup is an idempotent-separating homomorphic image of a proper inverse semigroup. The proof in [21] for this theorem uses completely different techniques. In this case $\mathfrak{T}$ is a free group, and the proofs for our lemmas simplify considerably.

The semigroup $\mathscr{D}$ is a $\mathcal{F}$-coextension of $D$, and so $\mathscr{D}$ and $D$ determine the same pseudo-semilattice. Therefore Lemma 2.3 can be applied: $\mathscr{D}$ is a rectangular band $I \times I$ of the semigroups $\mathscr{D}_{i j},(i, j) \in I \times I$. One can check that every $\mathscr{D}_{i j}$ contains a 
maximal inverse subsemigroup. Since $\mathscr{D}$ has injective structure mappings these inverse subsemigroups must be proper inverse semigroups. In particular, the semigroups $\mathscr{D}_{i i}, i \in I$, are proper inverse semigroups.

We summarize Theorem 2.1, Corollary 2.2, Remark 2.14 and Theorem 2.15 as follows.

THEOREM 2.16. Let $S$ be any pseudo-inverse semigroup. There exist pseudo-inverse semigroups $\mathbf{S}$ and $\mathcal{S}$, a homomorphism $\phi_{1}$ of $\mathcal{S}$ onto $\mathbf{S}$, and a homomorphism $\phi_{2}$ of $\mathbf{S}$ onto $S$ such that:

(i) $\delta$ is a pseudo-inverse semigroup which has injective structure mappings and $\phi_{1} \phi_{1}^{-1}$ is an idempotent-separating congruence on $\delta$.

(ii) The pseudo-semilattice $\left(\mathcal{E}, \omega^{l}, \omega^{r}\right)=\left(\mathbf{E}, \omega^{l}, \omega^{r}\right)$ which is determined by $\mathcal{S}$ and $\mathbf{S}$ is of the form

$$
\varepsilon=\mathbf{E}=\left(L_{\lambda}, R_{i} ; M_{i \lambda} ; \phi_{i \lambda}, \psi_{i \lambda} ; I, \Lambda\right)
$$

where the conditions (2.1) are satisfied.

(iii) The $\phi_{2} \phi_{2}^{-1}$-classes which contain idempotents are rectangular bands.

(iv) If $\gamma$ is the least primitive congruence on $\mathcal{S}$, then $\mathcal{S} / \gamma$ may be identified with a Rees matrix semigroup $\Re(G ; P ; I, \Lambda)$ such that

(a) every element of $\Re(G ; P ; I, \Lambda)$ which is a product of idempotents can be written in a unique way as a product $\left(p_{\lambda_{1} i_{1}}^{-1}\right)_{i_{1} \lambda_{1}} \cdots\left(p_{\lambda_{n} i_{n}}^{-1}\right)_{i_{n} \lambda_{n}}$ where $i_{k} \neq i_{k+1}$ and $\lambda_{k} \neq \lambda_{k+1}$ for all $1 \leqslant k \leqslant n-1$;

(b) if $x$ is any element of $S$ such that

$$
x \gamma^{\natural}=\left(p_{\lambda_{1} i_{1}}^{-1}\right)_{i_{1} \lambda_{1}} \cdots\left(p_{\lambda_{n} i_{n}}^{-1}\right)_{i_{n} \lambda_{n}}
$$

where $i_{k} \neq i_{k+1}$ and $\lambda_{k} \neq \lambda_{k+1}$ for all $1 \leqslant k \leqslant n-1$ then there exist idempotents $e_{i_{k} \lambda_{k}} \in\left(p_{\lambda_{k} i_{k}}^{-1}\right)_{i_{k} \lambda_{k}}\left(\gamma^{\natural}\right)^{-1}$ such that $x=e_{i_{1} \lambda_{1}} \cdots e_{i_{n} \lambda_{n}}$.

From this theorem it follows that the $\left(\phi_{1} \phi_{2}\right)\left(\phi_{1} \phi_{2}\right)^{-1}$-classes of $\mathcal{S}$ which contain idempotents form completely simple semigroups. In the terminology of [30] the congruence $\left(\phi_{1} \phi_{2}\right)\left(\phi_{1} \phi_{2}\right)^{-1}$ is strictly compatible with respect to the natural partial order on the pseudo-inverse semigroup $\delta$. In the terminology of [25], $\delta$ is a coextension of $S$ by completely simple semigroups. Thus, Theorem 2.16 states that, given any pseudo-inverse semigroup $S$, there exists a coextension of $S$ by completely simple semigroups which has injective structure mappings. Therefore Theorem 2.16 can be regarded as a generalization of McAlister's Theorem 2.4, Corollary 2.5 of [21], which states that, given any inverse semigroup $S$, there exists a $\mathcal{G}$-coextension of $S$ which has injective structure mappings. McAlister's Theorem 2.6 of [22] (the " $P$-theorem") provides a firm motivation for such construction: every inverse semigroup which has injective structure mappings is isomorphic to a $P$-semigroup $P(G, X, L)$, where the semilattice $L$ is an ideal of the partially ordered set $\mathscr{X}$ (see also [43]). The combination of McAlister's two results enables us to construct all inverse semigroups in terms of groups, semilattices and morphisms. We clearly need a $P$-theorem for pseudo-inverse semigroups. The next section will be devoted to this problem. 
3. The embedding. If $D$ is a pseudo-inverse semigroup which has injective structure mappings, then we know from [35, Proposition 3.3] that the pseudo-semilattice $\left(E(D), \omega^{l}, \omega^{r}\right)$ which is determined by $D$ is the disjoint union of its maximal subsemilattices. If the maximal subsemilattices are semilattices which have an identity, then the pseudo-semilattice which is determined by $D$ is of the form $E(D)=\left(L_{\lambda}, R_{i} ; M_{i \lambda} ; \phi_{i \lambda}, \psi_{i \lambda} ; I, \Lambda\right)$ by Result 2 . Let $\rho$ be the least primitive congruence on $D$. $D / \rho$ can be identified with the Rees matrix semigroup भ $(G ; P ; I, \Lambda)$. For any $(i, \lambda) \in I \times \Lambda, D_{i \lambda}$ will be the set of elements of $D$ which

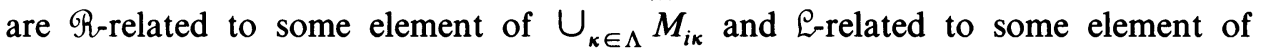
$\cup_{k \in I} M_{k \lambda}$. We already remarked in the introduction that $D$ is a rectangular band $I \times \Lambda$ of the semigroups $D_{i \lambda},(i, \lambda) \in I \times \Lambda$, and that $\rho$ induces a group congruence on each of the subsemigroups $D_{i \lambda},(i, \lambda) \in I \times \Lambda$ of $D$. If $e_{i \lambda} \in M_{i \lambda},(i, \lambda) \in I \times \Lambda$ is any idempotent of $D$ then $e_{i \lambda} \rho^{\natural}=\left(p_{\lambda i}^{-1}\right)_{i \lambda}$ is the identity of the maximal subgroup $G_{i \lambda}$ of $\mathfrak{N}(G ; P ; I, \Lambda)$.

In this section we shall suppose that $D$ is a pseudo-inverse semigroup which has injective structure mappings, that $E(D)=\left(L_{\lambda}, R_{i} ; M_{i \lambda} ; \phi_{i \lambda}, \psi_{i \lambda} ; I, \Lambda\right), \rho$ and $\mathscr{N}(G ; P ; I, \Lambda)$ are as described above, and that the following conditions are satisfied:

(3.1)(i) every element of the subsemigroup of $\Re(G ; P ; I, \Lambda)$ which is generated by the idempotents of $\mathscr{R}(G ; P ; I, \Lambda)$ can be written in a unique way as a product

$$
\left(p_{\lambda_{1} i_{1}}^{-1}\right)_{i_{1} \lambda_{1}}\left(p_{\lambda_{2} i_{2}}^{-1}\right)_{i_{2} \lambda_{2}} \cdots\left(p_{\lambda_{n} i_{n}}^{-1}\right)_{i_{n} \lambda_{n}}
$$

where $i_{m} \neq i_{m+1}, \lambda_{m} \neq \lambda_{m+1}$ for all $1 \leqslant m \leqslant n-1$,

(3.2)(ii) if $x$ is any element of $D$ such that

$$
x \rho^{\natural}=\left(p_{\lambda_{1} i_{1}}^{-1}\right)_{i_{1} \lambda_{1}} \cdots\left(p_{\lambda_{n} i_{n}}^{-1}\right)_{i_{n} \lambda_{n}}
$$

where $i_{m} \neq i_{m+1}, \lambda_{m} \neq \lambda_{m+1}$ for all $1 \leqslant m \leqslant n-1$, then there exist idempotents $e_{i_{m} \lambda_{m}} \in M_{i_{m} \lambda_{m}}, 1 \leqslant m \leqslant n$, such that $x=e_{i_{1} \lambda_{1}} e_{i_{2} \lambda_{2}} \cdots e_{i_{n} \lambda_{n}}$.

The semigroup $\delta$ which is obtained in $\$ 2$ satisfies these conditions. Therefore all the results which will be obtained in this section may be applied to $\mathcal{S}$. Remark that the conditions (3.1) and (3.2) imply that the preimage for $\rho^{\natural}$ of an idempotent $\left(p_{\lambda i}^{-1}\right)_{i \lambda}$ of $\Re(G ; P ; I, \Lambda)$ must be the maximal subsemilattice $M_{i \lambda}$ of $D$.

In [24, Theorem 2.5] it has been shown that $E(D)=\left(L_{\lambda}, R_{i} ; M_{i \lambda} ; \phi_{i \lambda}, \psi_{i \lambda} ; I, \Lambda\right)$ can be embedded as an order-ideal (with respect to the partial order $\omega$ ) into a pseudo-semilattice which is determined by an elementary rectangular band of proper inverse semigroups. The main result of this section indicates that $D$ itself can be embedded as a subsemigroup and as an order-ideal into an elementary rectangular band of proper inverse semigroups. Result 5 describes how such an elementary rectangular band of proper inverse semigroups can be constructed.

In the following lemma $\theta: D \rightarrow T_{E(D)}, x \rightarrow \theta_{x}$ denotes the fundamental regular representation of $D$.

LeMma 3.1. The mapping $\theta \times \rho^{\natural}: D \rightarrow T_{E(D)} \times(D / \rho), x \rightarrow\left(\theta_{x}, x \rho^{\natural}\right)$ is an isomorphism of $D$ onto a subsemigroup of $T_{E(D)} \times(D / \rho)$. 
Proof. Since $\theta \theta^{-1} \subseteq \mathcal{H}$ and $\rho \cap \Re=\iota_{D}$, we must have $\theta \theta^{-1} \cap \rho=\iota_{D}$. The lemma now follows.

We now recall part of a construction of $\$ 2$ in [24]. For any $\lambda \in \Lambda$, let $\bar{L}_{\lambda}$ be the semilattice $L_{\lambda}$ with an extra identity element $1_{\lambda}$ adjoined. Let $\left(\bar{M}_{i \lambda},(i, \lambda) \in I \times \Lambda\right)$ be an indexed family of pairwise disjoint semilattices such that, for every $(i, \lambda) \in I$ $\times \Lambda, \bar{M}_{i \lambda}$ is a semilattice which contains $M_{i \lambda}$ as an ideal, and such that $\bar{\phi}_{i \lambda}$ : $\bar{M}_{i \lambda} \rightarrow \bar{L}_{\lambda}$ is an isomorphism of $\bar{M}_{i \lambda}$ onto $\bar{L}_{\lambda}$ which extends $\phi_{i \lambda}$. We can always suppose that $R_{i} \cap\left(\cup_{\lambda \in \Lambda}\left(\bar{M}_{i \lambda} \backslash M_{i \lambda}\right)\right)=\square$ for all $i \in I$. For every $i \in I$, let $\bar{R}_{i}=$ $R_{i} \cup\left(\cup_{\lambda \in \Lambda}\left(\bar{M}_{i \lambda} \backslash M_{i \lambda}\right)\right)$, and for every $(i, \lambda) \in I \times \Lambda$, let $\bar{\psi}_{i \lambda}: \bar{M}_{i \lambda} \rightarrow \bar{R}_{i}$ be defined by

$$
\begin{aligned}
\bar{e}_{i \lambda} \bar{\psi}_{i \lambda} & =\bar{e}_{i \lambda} \quad \text { if } \bar{e}_{i \lambda} \in \bar{M}_{i \lambda} \backslash M_{i \lambda}, \\
& =\bar{e}_{i \lambda} \psi_{i \lambda} \quad \text { if } \bar{e}_{i \lambda} \in M_{i \lambda} .
\end{aligned}
$$

Define a partial order $\leqslant$ on $\bar{R}_{i}$ by the following: $\bar{e}_{i} \leqslant \bar{f}_{i}$ in $\bar{R}_{i}$ if and only if there exist $\lambda \in \Lambda, \bar{e}_{i \lambda}, \bar{f}_{i \lambda} \in \bar{M}_{i \lambda}$ such that $\bar{e}_{i \lambda} \leqslant \bar{f}_{i \lambda}$ in $\bar{M}_{i \lambda}$ and $\bar{e}_{i}=\bar{e}_{i \lambda} \bar{\psi}_{i \lambda}, \bar{f}_{i}=\bar{f}_{i \lambda} \bar{\psi}_{i \lambda} \cdot \bar{R}_{i}$ then becomes a semilattice, and for every $\lambda \in \Lambda, \bar{\psi}_{i \lambda}$ is an order-isomorphism of $\bar{M}_{i \lambda}$ onto a principal ideal of $\bar{R}_{i} ; \bar{\psi}_{i \lambda}$ extends $\psi_{i \lambda}$ for every $(i, \lambda) \in I \times \Lambda$. The pseudosemilattice $\bar{E}=\left(\bar{L}_{\lambda}, \bar{R}_{i} ; \bar{M}_{i \lambda} ; \bar{\phi}_{i \lambda}, \bar{\psi}_{i \lambda} ; I, \Lambda\right)$ contains $E(D)=\left(L_{\lambda}, R_{i} ; M_{i \lambda} ; \phi_{i \lambda}, \psi_{i \lambda}\right.$; $I, \Lambda)$ as an order-ideal.

Since $E(D)$ is an order-ideal of $\bar{E}$, the semigroup $T_{\bar{E}}$ contains the semigroup $T_{E(D)}$ as a subsemigroup. One can easily show that $T_{E(D)}$ is also an order-ideal of $T_{E}^{-}$with respect to the natural partial order on $T_{\bar{E}}^{-}$. Therefore $T_{\bar{E}}^{-} \times(D / \rho)$ contains $T_{E(D)} \times$ $(D / \rho)$ as a subsemigroup and as an order ideal, whereas the mapping $\theta \times \rho^{\natural}$ embeds $D$ isomorphically into $T_{\bar{E}}^{-} \times(D / \rho)$ by Lemma 3.1. Remark that $T_{E(D)}, T_{\bar{E}}$ and $(D / \rho)$ are pseudo-inverse semigroups. Since the class of pseudo-inverse semigroups is closed for taking direct products [29], it follows that $T_{E(D)} \times(D / \rho)$ and $T_{\bar{E}} \times(D / \rho)$ are pseudo-inverse semigroups.

It is well known that the mapping $E(D) \rightarrow E\left(T_{E(D)}\right), e_{i \lambda} \rightarrow \theta_{e_{i \lambda}}$ is an isomorphism of the pseudo-semilattice which is determined by $D$ onto the pseudo-semilattice which is determined by $T_{E(D)}$. This isomorphism can be extended to an isomorphism $\bar{E} \rightarrow E\left(T_{\bar{E}}^{-}\right), \bar{e}_{i \lambda} \rightarrow \theta_{\bar{e}_{i \lambda}}$ of the pseudo-semilattice $\bar{E}$ onto the pseudo-semilattice which is determined by $T_{\bar{E}}^{-}$.

For any $(i, \lambda) \in I \times \Lambda$, let $\bar{l}_{i \lambda}$ be the identity element of $\bar{M}_{i \lambda}$. Let $\bar{D}$ be the subset of $T_{\bar{E}}^{-} \times(D / \rho)$ which consists of the elements of the following two kinds:

(3.3)(i) the elements $\left(\theta_{i_{i \lambda}},\left(p_{\lambda i}^{-1}\right)_{i \lambda}\right),(i, \lambda) \in I \times \Lambda$,

(3.4)(ii) the elements of the form $\left(\alpha, a_{i \lambda}\right)$, where $\alpha \in T_{\bar{E}}^{-}, a \in G,(i, \lambda) \in I \times \Lambda$, such that for some $j \in I, \quad \mu \in \Lambda, \bar{e}_{i \mu} \in \bar{M}_{i \mu}, e_{j \mu} \in M_{j \mu}, x_{j \lambda} \in D_{j \lambda}$, with $\alpha \Re \theta_{\bar{e}_{i \mu}} \mathcal{L} \theta_{e_{j \mu}} \Re \theta_{x_{j \lambda}}$ in $T_{\bar{E}}^{-}, x_{j \lambda} \rho^{\natural}=\left(p_{\mu j}^{-1} p_{\mu i} a\right)_{j \lambda}$, we have $\theta_{\bar{e}_{i \mu}} \theta_{x_{j \lambda}}=\alpha$.

LEMMA 3.2. The set $E(\bar{D})$ of idempotents of $\bar{D}$ consists of the elements of the form $\left(\theta_{\bar{e}_{i \lambda}},\left(p_{\lambda i}^{-1}\right)_{i \lambda}\right),(i, \lambda) \in I \times \Lambda, \bar{e}_{i \lambda} \in \bar{M}_{i \lambda}$. The set $E(\bar{D})$ forms an order-ideal of the pseudo-semilattice of idempotents of $T_{\bar{E}}^{-} \times D / \rho$, and the mapping $\bar{E} \rightarrow E(\bar{D}), \bar{e}_{i \lambda} \rightarrow$ $\left(\theta_{\bar{e}_{i \lambda}},\left(p_{\lambda i}^{-1}\right)_{i \lambda}\right)$ is an isomorphism of pseudo-semilattices.

LEMMA 3.3. The set $\bar{D}$ is an order-ideal of $T_{\bar{E}}^{-} \times(D / \rho)$ with respect to the natural partial order on $T_{\bar{E}}^{-} \times(D / \rho)$. 
LEMMA 3.4. If $P$ and $Q$ are any elements of $\bar{D}$ such that $P Q$ is a trace product in $T_{\bar{E}}^{-} \times(D / \rho)$, then $P Q \in \bar{D}$.

Proof. We shall only consider the case where $P$ and $Q$ are both of type (ii). The other cases are easier. Suppose that $P=\left(\alpha, a_{i \lambda}\right)$, where for some $j \in I, \mu \in \Lambda$, $\bar{e}_{i \mu} \in \bar{M}_{i \mu}, e_{j \mu} \in M_{j \mu}, x_{j \lambda} \in D_{j \lambda}$ the conditions in (3.4) are satisfied, and $Q=\left(\beta, b_{n \nu}\right)$, where for some $h \in I, \eta \in \Lambda, \bar{f}_{n \eta} \in \bar{M}_{n \eta}, f_{h \eta} \in M_{h \eta}, y_{h \nu} \in D_{h \nu}$ with $\beta \Re \theta_{f_{n \eta}} \mathcal{L} \theta_{f_{h \eta}} \Re \theta_{y_{h \nu}}$ in $T_{E}^{-}, y_{h \nu} \rho^{\natural}=\left(p_{\eta h}^{-1} p_{\eta n} b\right)_{h \nu}$, we have $\theta_{f_{n \eta}}^{-} \theta_{y_{h \nu}}=\beta$. The idempotent of $T_{E}^{-1} \times(D / \rho)$

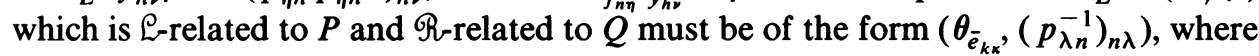
$\theta_{x_{j \lambda}} \mathcal{L} \mathcal{L} \theta_{\bar{e}_{k \kappa}} \Re \beta \Re \theta_{\bar{f}_{n \eta}}$. Since $x_{j \lambda} \mathcal{L} e_{l \lambda}$ in $D$ for some $l \in I, e_{l \lambda} \in M_{l \lambda}$, the foregoing implies $e_{I \lambda} \varrho \bar{e}_{k \kappa} \Re \bar{f}_{n \eta}$. Thus $\bar{e}_{k \kappa}=\bar{e}_{n \lambda}$, and so $P \mathcal{L}\left(\theta_{\bar{e}_{n \lambda}},\left(p_{\lambda n}^{-1}\right)_{n \lambda}\right) \Re Q$ in $T_{E}^{-} \times(D / \rho)$, where $P Q=\left(\alpha \beta,\left(a p_{\lambda n} b\right)_{i \nu}\right)$. From $\theta_{\bar{e}_{n \lambda}} \Re \theta_{f_{n \eta}}$ it follows that $\bar{e}_{n \lambda} \Re \bar{f}_{n \eta}$ in $\bar{E}$. The following two cases can occur: $\bar{e}_{n \lambda}=\bar{f}_{n \eta}$, or, $\bar{e}_{n \lambda} \neq \bar{f}_{n \eta}$ and $\bar{e}_{n \lambda} \in M_{n \lambda}, \bar{f}_{n \eta} \in M_{n \eta}$.

Case 1. $\bar{e}_{n \lambda}=\bar{f}_{n \eta}(\lambda=\eta)$. In this case $x_{j \lambda} \mathcal{L} f_{h \eta}=f_{h \lambda} \mathcal{R} y_{h \nu}$ in $D$, and $x_{j \lambda} y_{h \nu}$ is an element of $D_{j \nu}$ which is $R_{-}$-related to $e_{j \mu}$, such that

$$
\begin{aligned}
\left(x_{j \lambda} y_{h \nu}\right) \rho^{\natural} & =\left(p_{\mu j}^{-1} p_{\mu i} a\right)_{j \lambda}\left(p_{\eta h}^{-1} p_{\eta n} b\right)_{h \nu} \\
& =\left(p_{\mu j}^{-1} p_{\mu i} a\right)_{j \lambda}\left(p_{\lambda h}^{-1} p_{\lambda n} b\right)_{h \nu}=\left(p_{\mu j}^{-1} p_{\mu i} a p_{\lambda n} b\right)_{j \nu} .
\end{aligned}
$$

Moreover, $\alpha \beta \Re \theta_{\bar{e}_{i \mu}} \mathcal{L} \theta_{e_{j \mu}} \Re \theta_{x_{j \lambda} y_{h \nu}}$ in $T_{\bar{E}}^{-}$, and

$$
\boldsymbol{\theta}_{\bar{e}_{i \mu}} \theta_{x_{j \lambda} y_{h \nu}}=\theta_{\bar{e}_{i \mu}} \theta_{x_{j \lambda}} \theta_{y_{h \nu}}=\theta_{\bar{e}_{i \mu}} \theta_{x_{j \lambda} \lambda} \theta_{f_{n \eta}} \theta_{y_{h \nu}}=\alpha \beta \text {. }
$$

We conclude that $\left(\alpha \beta,\left(a p_{\lambda n} b\right)_{i \nu}\right)=P Q \in \bar{D}$.

Case 2. $\bar{e}_{n \lambda} \neq \bar{f}_{n \eta}$ and $\bar{e}_{n \lambda} \in M_{n \lambda}, \bar{f}_{n \eta} \in M_{n \eta}$. In this case $\bar{f}_{n \eta} y_{h \nu} \in D_{n \nu}$ and $\bar{e}_{n \lambda} \Re f_{n \eta} y_{h \nu}$ in $D$. Also $x_{j \lambda} \Re \bar{e}_{n \lambda}$ in $D$, and so $x_{j \lambda} \bar{f}_{n \eta} y_{h \nu} \in D_{j \nu}$ and $x_{j \lambda} f_{n \eta} y_{h \nu} \Re e_{j \mu}$ in $D$. Thus $\alpha \beta \Re \theta_{\bar{e}_{i \mu}} \mathcal{L} \theta_{e_{j \mu}} \Re \theta_{x_{j j} \bar{N}_{n \eta} y_{h \nu}}$ in $T_{E}^{-}$,

$$
\begin{aligned}
\left(x_{j \lambda} \bar{f}_{n \eta} y_{h \nu}\right) \rho^{\natural} & =\left(p_{\mu j}^{-1} p_{\mu i} a\right)_{j \lambda}\left(p_{\eta n}^{-1}\right)_{n \eta}\left(p_{\eta h}^{-1} p_{\eta n} b\right)_{h \nu} \\
& =\left(p_{\mu j}^{-1} p_{\mu i} a p_{\lambda n} p_{\eta n}^{-1} p_{\eta h} p_{\eta h}^{-1} p_{\eta n} b\right)_{j \nu} \\
& =\left(p_{\mu j}^{-1} p_{\mu i} a p_{\lambda n} b\right)_{j \nu}
\end{aligned}
$$

and

$$
\boldsymbol{\theta}_{\bar{e}_{i \mu}} \boldsymbol{\theta}_{x_{j \lambda} \bar{f}_{n \eta} y_{h \nu}}=\theta_{\bar{e}_{i \mu}} \boldsymbol{\theta}_{x_{j \lambda}} \theta_{\bar{f}_{n \eta}} \boldsymbol{\theta}_{y_{h \nu}}=\alpha \beta,
$$

and we conclude that $\left(\alpha \beta,\left(a p_{\lambda n} b\right)_{i v}\right)=P Q \in \bar{D}$.

LEMMA 3.5. $\bar{D}$ is a subsemigroup of $T_{E}^{-} \times(D / \rho)$, and the mapping $\theta \times \rho^{\natural}$ is an isomorphism of $D$ onto a subsemigroup of $\bar{D}$.

Proof. Let $P$ and $Q$ be any elements of $\bar{D}$. Then $P Q=P^{\prime} Q^{\prime}$ for some $P^{\prime}, Q^{\prime}$, with $P^{\prime} \leqslant P, Q^{\prime} \leqslant Q$, where $P^{\prime} Q^{\prime}$ is a trace product. By Lemmas 3.3 and 3.4 we conclude that $P^{\prime}, Q^{\prime}$ and $P^{\prime} Q^{\prime}$ belong to $\bar{D}$.

Let $x_{i \lambda} \in D_{i \lambda}$ be any element of $D$. It should be clear that $\left(\theta_{x_{i \lambda}}, x_{i \lambda} \rho^{\natural}\right) \in \bar{D}$. The proof now follows from Lemma 3.1.

LEMMA 3.6. $\bar{D}$ is a pseudo-inverse semigroup. 
Proof. All elements of type (i) of $\bar{D}$ are regular elements of $\bar{D}$ since they are idempotents. Let $P=\left(\alpha, a_{i \lambda}\right)$ be any element of type (ii) of $\bar{D}$, such that for some $j \in I, \mu \in \Lambda, \bar{e}_{i \mu} \in \bar{M}_{i \mu}, e_{j \mu} \in M_{j \mu}, x_{j \lambda} \in D_{j \lambda}$, the conditions in (3.4) are satisfied. Let $l \in I$ and $y_{l \mu} \in D_{l \mu}$ be an inverse of $x_{j \lambda}$ in $D$ such that $x_{j \lambda} y_{l \mu}=e_{j \mu}$ and

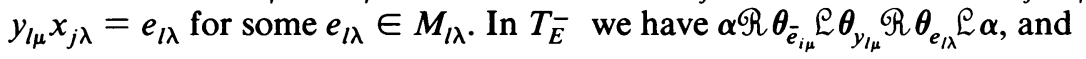

$$
\begin{aligned}
& \alpha \theta_{y_{l \mu}}=\theta_{\bar{e}_{i \mu}} \theta_{x_{j \lambda}} \theta_{y_{l \mu}}=\theta_{\bar{e}_{i \mu}} \theta_{e_{j \mu}}=\theta_{\bar{e}_{i \mu}}, \\
& \theta_{y_{l \mu}} \alpha=\theta_{y_{l \mu}} \theta_{\bar{e}_{i \mu}} \theta_{x_{j \lambda}}=\theta_{y_{l \mu}} \theta_{x_{j \lambda}}=\theta_{e_{l \lambda}} .
\end{aligned}
$$

Thus $\alpha$ and $\theta_{y_{l \mu}}$ are mutually inverse elements of $T_{\bar{E}}^{-}$. Obviously $y_{l \mu} \rho^{\natural}$ is the inverse in $G_{l \mu}$ of $x_{j \lambda} \rho^{\natural}=\left(p_{\mu j}^{-1} p_{\mu i} a\right)_{j \lambda}$. It is easy to compute that $y_{l \mu} \rho^{\natural}=\left(p_{\lambda l}^{-1} a^{-1} p_{\mu i}^{-1}\right)_{l \mu}$, and so

$$
\begin{aligned}
& a_{i \lambda}\left(y_{l \mu} \rho^{\natural}\right)=a_{i \lambda}\left(p_{\lambda l}^{-1} a^{-1} p_{\mu i}^{-1}\right)_{l \mu}=\left(a p_{\lambda l} p_{\lambda l}^{-1} a^{-1} p_{\mu i}^{-1}\right)_{i \mu}=\left(p_{\mu i}^{-1}\right)_{i \mu}, \\
& \left(y_{l \mu} \rho^{\natural}\right) a_{i \lambda}=\left(p_{\lambda l}^{-1} a^{-1} p_{\mu i}^{-1}\right)_{l \mu} a_{i \lambda}=\left(p_{\lambda l}^{-1} a^{-1} p_{\mu i}^{-1} p_{\mu i} a\right)_{l \lambda}=\left(p_{\lambda l}^{-1}\right)_{l \lambda} .
\end{aligned}
$$

One can readily check that $\left(\alpha, a_{i \lambda}\right)$ and $\left(\theta_{y_{l \mu}}, y_{l \mu} \rho^{\natural}\right)=\left(y_{l \mu}\right) \theta \times \rho^{\natural}$ are mutually inverse elements of $\bar{D}$. We conclude that $\bar{D}$ is a regular semigroup. By virtue of Lemma 3.2, $\bar{D}$ must be a pseudo-inverse semigroup.

LEMMA 3.7. $\bar{D}$ has injective structure mappings. The congruence $\sigma$ on $\bar{D}$ which is induced by the homomorphism

$$
\sigma^{\natural}: \bar{D} \rightarrow \operatorname{\Re (}(G ; P ; I, \Lambda), \quad\left(\theta_{1_{i \lambda}^{-}},\left(p_{\lambda i}^{-1}\right)_{i \lambda}\right) \rightarrow p_{\lambda i}^{-1}, \quad\left(\alpha, a_{i \lambda}\right) \rightarrow a_{i \lambda}
$$

is the least primitive congruence on $\bar{D}$, and $\Re(G ; P ; I, \Lambda)$ is the greatest completely simple homomorphic image of $\bar{D}$. The pseudo-inverse semigroup $\bar{D}$ satisfies conditions (3.1) and (3.2).

Proof. Since $\bar{D}$ is a subsemigroup of $T_{\bar{E}}^{-} \times(D / \rho)$, the above considered mapping $\sigma^{\natural}$ is a homomorphism, and $\sigma$ is a primitive congruence on $\bar{D}$. Thus $\sigma \supseteq \leqslant \circ(\leqslant)^{-1}$, where $\leqslant \circ(\leqslant)^{-1}$ is known to be the least primitive congruence on $\bar{D}[\mathbf{3 0}]$.

Let $P$ and $T$ be any elements of $\bar{D}$ such that $T \leqslant P$ in $\bar{D}$. If $P=\left(\theta_{\overline{1}_{i \lambda}},\left(p_{\lambda i}^{-1}\right)_{i \lambda}\right)$, then of course $T=\left(\theta_{\bar{e}_{i \lambda}},\left(p_{\lambda i}^{-1}\right)_{i \lambda}\right)$ for some $\bar{e}_{i \lambda} \in \bar{M}_{i \lambda}$. Let us now suppose that $P=\left(\alpha, a_{i \lambda}\right)$ is of type (ii), where for some $j \in I, \mu \in \Lambda, \bar{e}_{i \mu} \in \bar{M}_{i \mu}, e_{j \mu} \in M_{i \mu}$, $x_{j \lambda} \in D_{j \lambda}$ the conditions in (3.4) are satisfied. Clearly $P \Re\left(\theta_{\bar{e}_{i \mu}},\left(p_{\mu i}^{-1}\right)_{i \mu}\right)$ and from $T \leqslant P$ it follows that $T \Re\left(\theta_{\bar{f}_{i \mu}},\left(p_{\mu i}^{-1}\right)_{i \mu}\right)$ for some $\bar{f}_{i \mu} \in \bar{M}_{i \mu}$, with $\bar{f}_{i \mu} \omega \bar{e}_{i \mu}$, and

$$
T=\left(\theta_{f_{i \mu}},\left(p_{\mu i}^{-1}\right)_{i \mu}\right)\left(\alpha, a_{i \lambda}\right)=\left(\theta_{f_{i \mu}} \alpha, a_{i \lambda}\right) \text {. }
$$

Thus $P \sigma^{\natural}=T \sigma^{\natural}$ in both cases, and we conclude that $\sigma=\leqslant \circ(\leqslant)^{-1}$.

We shall now make use of Result 4 to show that $\bar{D}$ has injective structure mappings. It is therefore sufficient to show that the preimage of any idempotent $\left(p_{\lambda i}^{-1}\right)_{i \lambda}$ of $\Re(G ; P ; I, \Lambda)$ for $\sigma^{\natural}$ is the $\sigma$-class consisting of the elements of $\bar{M}_{i \lambda}$. This will follow from the following considerations.

We know that the semigroup $D / \rho=\bar{D} / \sigma$ satisfies conditions (3.1) and (3.2). Let us now consider any element of type (ii) of $\bar{D}$ which is of the form $\left(\alpha, a_{i_{1} \lambda_{n}}\right)$, where $a_{i_{1} \lambda_{n}}=\left(p_{\lambda_{1} i_{1}}^{-1}\right)_{i_{1} \lambda_{1}} \cdots\left(p_{\lambda_{n} i_{n}}^{-1}\right)_{i_{n} \lambda_{n}}$, with $i_{m} \neq i_{m+1}, \lambda_{m} \neq \lambda_{m+1}$ for all $1 \leqslant m \leqslant n-1$. 
There exist $i \in I, \lambda \in \Lambda, \bar{e}_{i_{1} \lambda} \in \bar{M}_{i_{1} \lambda}, \quad e_{i \lambda} \in M_{i \lambda}, y_{i \lambda_{n}} \in D_{i \lambda_{n}}$ such that $\alpha \Re \theta_{\bar{e}_{i, \lambda}} \mathcal{L} \theta_{e_{i \lambda}} \Re \theta_{y_{i \lambda_{n}}} \Re \theta_{y_{i \lambda_{n}}}$ in $T_{\bar{E}}^{-}$,

$$
\begin{aligned}
y_{i \lambda_{n}} \rho^{\natural} & =\left(p_{\lambda i}^{-1} p_{\lambda_{i}} p_{\lambda_{1} i_{1}}^{-1} p_{\lambda_{1} i_{2}} \cdots p_{\lambda_{n} i_{n}}^{-1}\right)_{i \lambda_{n}} \\
& =\left(p_{\lambda i}^{-1}\right)_{i \lambda}\left(p_{\lambda_{1} i_{1}}^{-1}\right)_{i_{1} \lambda_{1}} \cdots\left(p_{\lambda_{n} i_{n}}^{-1}\right)_{i_{n} \lambda_{n}},
\end{aligned}
$$

and

$$
\boldsymbol{\alpha}=\boldsymbol{\theta}_{\bar{e}_{i_{1} \lambda}} \boldsymbol{\theta}_{y_{i \lambda}} .
$$

There are three cases to consider: (1) $i=i_{1}$, (2) $\lambda=\lambda_{1}$ and (3) $i \neq i_{1}$ and $\lambda \neq \lambda_{1}$.

Case 1. $i=i_{1}$. In this case $\alpha=\theta_{y_{i_{1} \lambda_{n}}}$ and $\left(\alpha, a_{i_{1} \lambda_{n}}\right)=\left(y_{i_{1} \lambda_{n}}\right) \theta \times \rho^{\natural}$. Since $D$ satisfies conditions (3.1) and (3.2), there exist $e_{i_{m} \lambda_{m}} \in M_{i_{m} \lambda_{m}}, m=1, \ldots, n$, such that $y_{i_{1} \lambda_{n}}=e_{i_{1} \lambda_{1}} \cdots e_{i_{n} \lambda_{n}}$ in $D$. Consequently

$$
\left(\alpha, a_{i_{1} \lambda_{n}}\right)=\left(\theta_{e_{i_{1} \lambda_{1}}},\left(p_{\lambda_{1} i_{1}}^{-1}\right)_{i_{1} \lambda_{1}}\right) \cdots\left(\theta_{e_{i_{n} \lambda_{n}}},\left(p_{\lambda_{n} i_{n}}^{-1}\right)_{i_{n} \lambda_{n}}\right)
$$

where, for every $m=1, \ldots, n,\left(\theta_{e_{i_{m} \lambda_{m}}},\left(p_{\lambda_{m} i_{m}}^{-1}\right)_{i_{m} \lambda_{m}}\right)$ is an idempotent of $\bar{D}$ such that

$$
\left(\theta_{e_{i_{m} \lambda_{m}}},\left(p_{\lambda_{m} i_{m}}^{-1}\right)_{i_{m} \lambda_{m}}\right) \sigma^{\natural}=\left(p_{\lambda_{m} i_{m}}^{-1}\right)_{i_{m} \lambda_{m}} \text {. }
$$

Case 2. $\lambda=\lambda_{1}$. In this case $y_{i \lambda_{n}} \rho^{\natural}=\left(p_{\lambda_{1} i}^{-1}\right)_{i \lambda_{1}}\left(p_{\lambda_{2} i_{2}}^{-1}\right)_{i_{2} \lambda_{2}} \cdots\left(p_{\lambda_{n} i_{n}}^{-1}\right)_{i_{n} \lambda_{n}}$. Clearly $\lambda=\lambda_{1} \neq \lambda_{2}$. We consider two subcases: (a) $i=i_{2}$ and (b) $i \neq i_{2}$.

Case 2(a). $\lambda=\lambda_{1}, i=i_{2}$. In this case $y_{i_{2} \lambda_{n}} \rho^{\natural}=\left(p_{\lambda_{2} i_{2}}^{-1}\right)_{i_{2} \lambda_{2}} \cdots\left(p_{\lambda_{n} i_{n}}^{-1}\right)_{i_{n} \lambda_{n}}$, and so there exist $e_{i_{m} \lambda_{m}} \in M_{i_{m} \lambda_{m}}, m=2, \ldots, n$, such that $y_{i_{2} \lambda_{n}}=e_{i_{2} \lambda_{2}} \cdots e_{i_{n} \lambda_{n}}$. Consequently

$$
\left(\alpha, a_{i_{1} \lambda_{n}}\right)=\left(\theta_{\bar{e}_{i_{1} \lambda_{1}}},\left(p_{\lambda_{1} i_{1}}^{-1}\right)_{i_{1} \lambda_{1}}\right)\left(\theta_{e_{i_{2} \lambda_{2}}},\left(p_{\lambda_{2} i_{2}}^{-1}\right)_{i_{2} \lambda_{2}}\right) \cdots\left(\theta_{e_{i_{n} \lambda_{n}}},\left(p_{\lambda_{n} i_{n}}^{-1}\right)_{i_{n} \lambda_{n}}\right) .
$$

Case 2(b). $\lambda=\lambda_{1}, i \neq i_{2}$. In this case $y_{i \lambda_{n}} \rho^{\natural}=\left(p_{\lambda i}^{-1}\right)_{i \lambda}\left(p_{\lambda_{2} i_{2}}^{-1}\right)_{i_{2} \lambda_{2}} \cdots\left(p_{\lambda_{n} i_{n}}^{-1}\right)_{i_{n} \lambda_{n}}$, and so there exist $e_{i_{m} \lambda_{m}} \in M_{i_{m} \lambda_{m}}, m=2, \ldots, n$, such that $y_{i \lambda_{n}}=e_{i \lambda} e_{i_{2} \lambda_{2}} \cdots e_{i_{n} \lambda_{n}}$. Then

$$
\alpha=\theta_{\bar{e}_{i_{1} \lambda}} \boldsymbol{\theta}_{e_{i \lambda}} \theta_{e_{i_{2} \lambda_{2}}} \cdots \theta_{e_{i_{n} \lambda_{n}}}=\theta_{\bar{e}_{i_{1} \lambda_{1}}} \theta_{e_{i_{2} \lambda_{2}}} \cdots \theta_{e_{i_{n} \lambda_{n}}},
$$

thus again

$$
\left(\alpha, a_{i_{1} \lambda_{n}}\right)=\left(\boldsymbol{\theta}_{\bar{e}_{i_{1} \lambda_{1}}},\left(p_{\lambda_{1} i_{1}}^{-1}\right)_{i_{1} \lambda_{1}}\right)\left(\theta_{e_{i_{2} \lambda_{2}}},\left(p_{\lambda_{2} i_{2}}^{-1}\right)_{i_{2} \lambda_{2}}\right) \ldots\left(\theta_{e_{i_{n} \lambda_{n}}},\left(p_{\lambda_{n} i_{n}}^{-1}\right)_{i_{n} \lambda_{n}}\right) .
$$

Case 3. $i \neq i_{1}, \lambda \neq \lambda_{1}$. In this case $y_{i \lambda_{n}} \rho^{\natural}=\left(p_{\lambda i}^{-1}\right)_{i \lambda}\left(p_{\lambda_{1} i_{1}}^{-1}\right)_{i_{1} \lambda_{1}} \cdots\left(p_{\lambda_{n} i_{n}}^{-1}\right)_{i_{n} \lambda_{n}}$, and so there exist $e_{i_{m} \lambda_{m}} \in M_{i_{m} \lambda_{m}}, m=1, \ldots, n$, such that $y_{i \lambda_{n}}=e_{i \lambda} e_{i_{1} \lambda_{1}} \cdots e_{i_{n} \lambda_{n}}$. Then

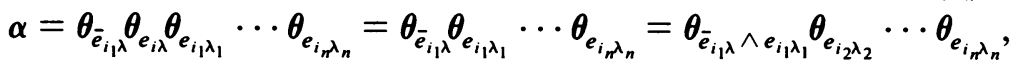

where $\bar{e}_{i_{1} \lambda} \wedge e_{i_{1} \lambda_{1}} \in \bar{M}_{i_{1} \lambda_{1}}$, and so

$$
\left(\alpha, a_{i_{1} \lambda_{n}}\right)=\left(\theta_{\bar{e}_{i_{1} \lambda} \wedge e_{i_{1} \lambda_{1}}},\left(p_{\lambda_{1} i_{1}}^{-1}\right)_{i_{1} \lambda_{1}}\right)\left(\theta_{e_{i_{2} \lambda_{2}}},\left(p_{\lambda_{2} i_{2}}^{-1}\right)_{i_{2} \lambda_{2}}\right) \cdots\left(\theta_{e_{i_{n} \lambda_{n}}},\left(p_{\lambda_{n} i_{n}}^{-1}\right)_{i_{n} \lambda_{n}}\right) .
$$

We conclude that $\bar{D}$ has injective structure mappings and that $\bar{D}$ satisfies conditions (3.1) and (3.2). 
LEMMA 3.8. The semigroup $D \theta \times \rho^{\natural}$ consists of the elements $\left(\alpha, a_{i \lambda}\right)$ of type (ii) of $\bar{D}$ for which there exist some idempotents $e_{i \kappa}, f_{k \lambda}$ in $E(D)$ such that

$$
\left(\theta_{e_{i \kappa}},\left(p_{\kappa i}^{-1}\right)_{i \kappa}\right) \Re\left(\alpha, a_{i \lambda}\right) \mathcal{E}\left(\theta_{f_{k \lambda}},\left(p_{\lambda k}^{-1}\right)_{k \lambda}\right)
$$

in $\bar{D}$. The subsemigroup $D \theta \times \rho^{\natural}$ of $\bar{D}$ is an order-ideal of $\bar{D}$.

THEOREM 3.9. The pseudo-inverse semigroup $D$ which has injective structure mappings and which satisfies conditions (3.1) and (3.2) can be embedded as a subsemigroup and as an order-ideal in an elementary rectangular band of proper inverse semigroups $D^{\prime}$ in such a way that $D$ consists of the elements $x$ of $D^{\prime}$ for which there exist some

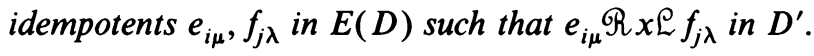

Proof. Let us consider a sequence of pseudo-semilattices

$$
E(D)=E_{0}, E_{1}, \ldots, E_{k}, E_{k+1}, \ldots,
$$

$E_{k}=\left(L_{\lambda}^{(k)}, R_{i}^{(k)} ; M_{i \lambda}^{(k)} ; \phi_{i \lambda}^{(k)}, \psi_{i \lambda}^{(k)} ; I, \Lambda\right)$, where $E_{1}=\bar{E}$, where $E_{k+1}$ is constructed from $E_{k}$ in the same way as $\bar{E}$ is constructed from $E(D)$ if $k$ is even, and where $E_{k+1}$ is constructed from $E_{k}$ in a dual way as $\bar{E}$ is constructed from $E(D)$ if $k$ is odd. Using induction, we can show by Lemmas 3.2-3.8 that there exists a sequence of pseudo-inverse semigroups $D=D_{0}, D_{1}, \ldots, D_{k}, D_{k+1}, \ldots$ such that, for every $k \in \mathbf{N}$,

(i) $E_{k}$ is the pseudo-semilattice $\left(E\left(D_{k}\right), \omega^{l}, \omega^{r}\right)$ which is determined by $D_{k}$;

(ii) $D_{k}$ is a pseudo-inverse semigroup which has injective structure mappings, and the greatest completely simple homomorphic image of $D_{k}$ may be identified with $D / \rho=\mathfrak{R}(G ; P ; I, \Lambda)$, such that conditions (3.1) and (3.2) are satisfied;

(iii) $D_{k}$ is a subsemigroup and an order-ideal of $D_{k+1}$;

(iv) $D_{k}$ consists of the elements $x^{(k)}$ of $D_{k+1}$ for which there exist some idempotents $e_{i \mu}^{(k)}, f_{j \lambda}^{(k)}$ in $E_{k}$ such that $e_{i \mu}^{(k)} \Re x^{(k)} \varrho f_{j \lambda}^{(k)}$ in $D_{k+1}$.

Let $D^{\prime}=\cup_{k=0}^{\infty} D_{k}$, and define a multiplication on $D^{\prime}$ in the obvious way: if $x, y \in D^{\prime}$, then $x, y \in D_{n}$ for some natural number $n$, and the product $x y$ of $x$ and $y$ in $D^{\prime}$ coincides with the product of $x$ and $y$ in $D_{n}$. By this $D^{\prime}$ becomes a pseudo-inverse semigroup. Let $E^{\prime}$ be the pseudo-semilattice which is determined by $D^{\prime}$. The pseudo-semilattice $E^{\prime}$ is the direct limit (in the sense of [24, Theorem 2.5]) of the pseudo-semilattices $E_{0}, \ldots, E_{k}, E_{k+1}, \ldots D^{\prime}$ contains $D$ as a subsemigroup and as an order-ideal. $D^{\prime}$ has injective structure mappings. Furthermore, $D$ consists of the elements $x$ of $D^{\prime}$ for which there exist some idempotents $e_{i \mu}, f_{j \lambda}$ in $E(D)$ such that $e_{i \mu} \Re x \varrho f_{j \lambda}$ in $D^{\prime}$.

From the proof of Theorem 2.5 of [24] it follows that $E^{\prime}$ is the pseudo-semilattice which is determined by an elementary rectangular band of inverse semigroups. From $\S \S 3.1$ and 3.2 of [35] it follows that every pseudo-inverse semigroup which determines the pseudo-semilattice $E^{\prime}$ must be an elementary rectangular band of inverse semigroups. In particular, $D^{\prime}$ is a pseudo-inverse semigroup which has injective structure mappings, and which is an elementary rectangular band $I \times \Lambda$ of inverse semigroups $D_{i \lambda}^{\prime},(i, \lambda) \in I \times \Lambda$. Clearly the maximal inverse subsemigroups $D_{i \lambda}^{\prime},(i, \lambda) \in I \times \Lambda$, of $D^{\prime}$, must have injective structure mappings too, and therefore they are proper inverse semigroups. This completes the proof of the theorem. 
From Theorem 2.16 and the foregoing theorem it follows that every pseudoinverse semigroup divides some pseudo-inverse semigroup which is an elementary rectangular band of proper inverse semigroups. This fact will be exploited in $\S 4$.

\section{Main results.}

THEOREM 4.1. Let $\mathfrak{X}$ be a partially ordered set which contains the semilattice L as a subsemilattice and as an order-ideal. Let $G$ be a group which acts on $\mathcal{X}$ (on the left) as a group of order automorphisms. Let $I$ be an index set, let $\left(A_{i}, i \in I\right)$ be a family of elements of $L$, and let $\left(p_{j i},(i, j) \in I \times I\right)$ be a family of elements of $G$ which have actions on $\mathcal{X}$ that induce automorphisms on L. Let $S$ be the set which consists of the elements $(A, g)_{i j}, A \in L, g \in G,(i, j) \in I \times I$, where $A \leqslant A_{i}$ and $p_{j j}^{-1} g^{-1} A \leqslant A_{j}$. Define a multiplication on $S$ by

$$
(A, g)_{i j}(B, h)_{m n}=\left(A \wedge g p_{j m} B, g p_{j m} h\right)_{i n} .
$$

Then $S$ becomes a pseudo-inverse semigroup.

Let $\rho$ be a strictly compatible congruence on $S$. Then $S / \rho$ is a pseudo-inverse semigroup, and conversely, any pseudo-inverse semigroup can be so constructed.

Proof. Let us consider the elementary rectangular band of proper inverse semigroups $\mathscr{N}(P(G, \mathfrak{X}, L) ; P ; I, I)$ (see Result 5$)$. We show that $S$ is a regular subsemigroup of $\mathfrak{T}(P(G, \mathcal{X}, L) ; P ; I, I)$. For every $(A, g)_{i j}$ of $\mathfrak{T}(P(G, \mathcal{X}, L) ; P$; $I, I)$ we have

$$
\left(p_{j j}^{-1} g^{-1} A, p_{j j}^{-1}\right)_{j j} \mathcal{L}(A, g)_{i j} \Re\left(A, p_{i i}^{-1}\right)_{i i}
$$

where $\left(p_{j j}^{-1} g^{-1} A, p_{j j}^{-1}\right)_{j j}$ and $\left(A, p_{i i}^{-1}\right)_{i i}$ are idempotents which belong to the maximal subsemilattices $\left\{\left(X, p_{j j}^{-1}\right)_{j j} \mid X \in L\right\}$ and $\left\{\left(X, p_{i i}^{-1}\right)_{i i} \mid X \in L\right\}$ respectively. Therefore, an element $(A, g)_{i j}$ of $\Re(P(G, X, L) ; P ; I, I)$ belongs to $S$ if and only if $(A, g)_{i j}$ is in the intersection of the principal left ideal which is generated by $\left(A_{j}, p_{j j}^{-1}\right)_{j j}$ and the principal right ideal which is generated by $\left(A_{i}, p_{i i}^{-1}\right)_{i i}$. This shows that $S$ is a subsemigroup of $\Re(P(G, \mathcal{X}, L) ; P ; I, I)$. If $(A, g)_{i j}$ belongs to $S$, then the inverse $\left(p_{j j}^{-1} g^{-1} A, p_{j j}^{-1} g^{-1} p_{i i}^{-1}\right)_{j i}$ of $(A, g)_{i j}$ also belongs to $S$, and so $S$ is a regular semigroup. $S$ is a pseudo-inverse semigroup since $S$ is a regular subsemigroup of a pseudo-inverse semigroup. The direct part of the proof follows, since the class of pseudo-inverse semigroups is closed for taking homomorphic images.

Let us now suppose that $S$ is any pseudo-inverse semigroup. Let $\mathcal{S}$ be as in Theorem 2.16. There exists a strictly compatible congruence $\rho$ on $\mathcal{S}$ such that $S$ is isomorphic to $\delta / \rho$. We know that $\delta$ can be embedded as a subsemigroup and as an order-ideal in an elementary rectangular band of proper inverse semigroups, in the way prescribed by Theorem 3.9. Since the pseudo-semilattice $\mathcal{E}=\left(L_{i}, R_{i} ; M_{i j}\right.$; $\left.\phi_{i j}, \psi_{i j} ; I, I\right)$ which is determined by $\delta$ satisfies the conditions $(2.1)$, this elementary rectangular band of proper inverse semigroups is of the form $\mathfrak{R}(P(G, \mathcal{X}, L) ; P$; $I, I)$, and the identity elements of the maximal subsemilattices $M_{i i}, i \in I$, are of the form $\left(A_{i}, p_{i i}^{-1}\right)_{i i}, i \in I$. Theorem 3.9 states that $\delta$ consists of the elements $(A, g)_{i j}$ of $\Re(P(G, \mathcal{X}, L) ; P ; I, I)$ for which there exist $m, n \in I$, and idempotents $\left(X, p_{n i}^{-1}\right)_{i n}$ $\in M_{i n},\left(Y, p_{j m}^{-1}\right)_{m j} \in M_{m j}$ such that

$$
\left(X, p_{n i}^{-1}\right)_{i n} \Re(A, g)_{i j} \mathcal{L}\left(Y, p_{j m}^{-1}\right)_{m j}
$$


in $\mathfrak{R}(P(G, \mathfrak{X}, L) ; P ; I, I)$. Since the pseudo-semilattice which is determined by $\delta$ satisfies conditions $(2.1), \mathcal{S}$ consists of the elements $(A, g)_{i j}$ of $\Re(P(G, \mathcal{X}, L) ; P$; $I, I)$ where $(A, g)_{i j} \mathcal{R}\left(A, p_{i i}^{-1}\right)_{i i} \leqslant\left(A_{i}, p_{i i}^{-1}\right)_{i i}$ and $(A, g)_{i j} \mathcal{L}\left(p_{j j}^{-1} g^{-1} A, p_{j j}^{-1}\right)_{j j} \leqslant$ $\left(A_{j}, p_{j j}^{-1}\right)_{j j}$. Thus, an element $(A, g)_{i j}$ of $\Re(P(G, \mathfrak{X}, L) ; P ; I, I)$ belongs to $\mathcal{S}$ if and only if $A \leqslant A_{i}$ and $p_{j j}^{-1} g^{-1} A \leqslant A_{j}$ in $L$. Therefore the converse part of the theorem holds.

THEOREM 4.2. Every pseudo-inverse semigroup divides a pseudo-inverse semigroup গ $(P(G, \bar{L}, \bar{L}) ; P ; I, I)$ which is a semidirect product of the completely simple semigroup $\Re(G ; P ; I, I)$ and the semilattice $\bar{L}$.

Proof. From the foregoing theorem it follows that every pseudo-inverse semigroup divides an elementary rectangular band of proper inverse semigroups IR $(P(G, X, L) ; P ; I, I)$. From Theorem 1.3 of [31] we know that there exist a semilattice $\bar{L}$ which contains $L$ as a subsemilattice, and a $P$-semigroup $P(G, \bar{L}, \bar{L})$ which is a semidirect product of the group $G$ and the semilattice $\bar{L}$, such that the inclusion mapping $P(G, \mathcal{X}, L) \rightarrow P(G, \bar{L}, \bar{L}),(A, g) \rightarrow(A, g)$ embeds $P(G, X, L)$ isomorphically into $P(G, \bar{L}, \bar{L})$. Clearly the inclusion mapping

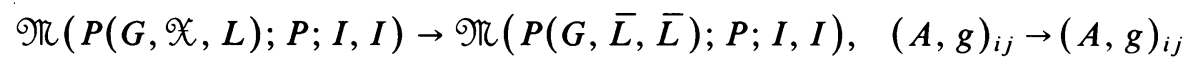

embeds $\Re(P(G, \mathcal{X}, L) ; P ; I, I)$ isomorphically into the semigroup $\Re(P(G, \bar{L}, \bar{L})$; $P ; I, I)$, which is a semidirect product of the completely simple semigroup $\Re(G ; P$; $I, I)$ and the semilattice $\bar{L}$.

REMARKS 4.3. We showed that every pseudo-inverse semigroup $S$ divides an elementary rectangular band of proper inverse semigroups of the form TL $(P(G, \mathfrak{X}, L) ; P ; I, I)$. If we take a pseudo-inverse semigroup $S$ of some particular kind, then it can occur that we can handle the case in a more economical way, and that we can vary the division procedure and thereby obtain sharper results. In [24] we already made the remark that every normal band divides a band which is the direct product of a semilattice and a rectangular band; moreover, every orthodox pseudo-inverse semigroup $S$ (i.e. generalized inverse semigroup [48]) divides a pseudo-inverse semigroup which is the direct product of an inverse semigroup and a rectangular band [24, Theorem 4.8]. Every fundamental pseudo-inverse semigroup $S$ divides a fundamental pseudo-inverse semigroup which is an elementary rectangular band of fundamental inverse semigroups [24, Theorem 4.2]. The results of $\S 4$ of [24] indicate that several simplifications will be possible if the pseudo-inverse semigroup from which we start is combinatorial, or completely semisimple.

Remark that a matrix representation for primitive regular semigroups can immediately be obtained from Theorem 4.1: in this case $L$ must be an atomic lattice, and the elements $A_{i}, i \in I$, atoms of $L$. The completely 0 -simple semigroups arise from the case where $G$ acts transitively on the atoms $A_{i}, i \in I$.

\section{REFERENCES}

1. K. Byleen, The structure of regular and inverse semigroups, Dissertation, Univ. of Nebraska-Lincoln, 1977.

2. Spirals of idempotents from P-semigroups, Semigroup Forum 17 (1979), 95-100. 6-26.

3. K. Byleen, J. Meakin and F. Pastijn, The fundamental four-spiral semigroup, J. Algebra 54 (1978), 
4. __ Building bisimple idempotent-generated semigroups, J. Algebra 65 (1980), 60-83.

5. A. H. Clifford, The fundamental representation of a regular semigroup, Preprints and Lecture Notes in Mathematics, Tulane Univ., 1974.

6. $\ldots$ The fundamental representation of a regular semigroup, Semigroup Forum 10 (1975), 84-92.

7. A. H. Clifford and G. B. Preston, The algebraic theory of semigroups, Vols. I, II, Math. Surveys, no. 7, Amer. Math. Soc., Providence, R. I., 1961, 1967.

8. R. L. Gantos, Semilattices of bisimple inverse semigroups, Quart. J. Math. Oxford 22 (1971), 379-393.

9. T. E. Hall, On regular semigroups whose idempotents form a subsemigroup, Bull. Austral. Math. Soc. 1 (1969), 195-208.

10. D. W. Hardy and Y. Tirasupa, Semilattices of proper inverse semigroups, Semigroup Forum 13 (1976), 29-36.

11. J. M. Howie, An introduction to semigroup theory, Academic Press, London, 1976

12. G. Lallement, Congruences et équivalences de Green sur un demi-groupe régulier, C. R. Acad. Sci. Paris Sér. A. 262 (1966), 613-616.

13. __ Demi-groupes réguliers, Ann. Mat. Pura Appl. 77 (1967), 43-130.

14. Structure theorems for regular semigroups, Semigroup Forum 4 (1972), 95-123.

15. __ Some remarks on the four-spiral semigroup, Semigroup Forum 18 (1979), 341-345.

16. G. Lallement and M. Petrich, Décompositions I-matricielles d'un demi-groupe, J. Math. Pures Appl. 45 (1966), 67-117.

17. , A generalization of the Rees theorem in semigroups, Acta Sci. Math. (Szeged) 30 (1969), $113-132$

18. __ Structure d' une classe de demi-groupes réguliers, J. Math. Pures Appl. 48 (1969), 345-397.

19. ___ Extensions of a Brandt semigroup by another, Canad. J. Math. 22 (1970), 974-983.

20. E. S. Ljapin, Semigroups, Amer. Math. Soc., Providence, R. I., 1974.

21. D. B. McAlister, Groups, semilattices and inverse semigroups, Trans. Amer. Math. Soc. 192 (1974), 227-244.

22. __ Groups, semilattices and inverse semigroups. II, Trans. Amer. Math. Soc. 196 (1974), $351-370$.

23. J. Meakin, The structure mappings on a regular semigroup, Proc. Edinburgh Math. Soc. 21 (1978), $135-142$.

24. J. Meakin and F. Pastijn, The structure of pseudo-semilattices, Algebra Universalis 13 (1981), 355-372.

25. J. Meakin and K. S. S. Nambooripad, Coextensions of regular semigroups by rectangular bands. I, Trans. Amer. Math. Soc. 269 (1982), 197-224.

26. K. S. S. Nambooripad, Structure of regular semigroups, Thesis, Univ. of Kerala, 1973.

27. (1975), 354-363.

28. __ Structure of regular semigroups. II. The general case, Semigroup Forum 9 (1975), 364-371.

29. ___ Pseudo-semilattices and biordered sets, I, Simon Stevin 55 (1981), 103-110.

30. __ The natural partial order on a regular semigroup, Proc. Edinburgh Math. Soc. (to appear)

31. L. O'Carroll, Embedding theorems for proper inverse semigroups, J. Algebra 42 (1976), 26-40.

32. F. Pastijn, Embedding semigroups in semibands, Semigroup Forum 14 (1.977), 247-263.

33. On Schein's structure theorem for proper bands of monoids (submitted).

34. __ Maximal subsemilattices of simple pseudo-inverse semigroups, Simon Stevin 53 (1979), $121-130$

35. __ Rectangular bands of inverse semigroups, Simon Stevin 56 (1982), 1-97.

36. M. Petrich, A construction and a classification of bands, Math. Nachr. 48 (1971), 263-274.

37. , The structure of completely regular semigroups, Trans. Amer. Math. Soc. 189 (1974), 211-236.

38. M. Petrich, Lectures in semigroups, Wiley, London, 1977.

39. G. B. Preston, Matrix representations of inverse semigroups, J. Austral. Math. Soc. 2 (1969), 29-61.

40. D. Rees, On semi-groups, Proc. Cambridge Philos. Soc. 36 (1940), 387-400.

41. B. M. Schein, Pseudo-semilattices and pseudo-lattices, Izv. Vyš̌. Učebn. Zaved Matematika 2(117) (1972), 81-94. (Russian)

42. __ Bands of monoids, Acta Sci. Math. (Szeged) 36 (1974), 145-154.

43. __ A new proof for the McAlister "P-Theorem", Semigroup Forum 10 (1975), 185-188. 
44. O. Steinfeld, On semigroups which are unions of completely 0-simple semigroups, Czechoslovak Math. J. 16 (91) (1966), 63-69.

45. A. K. Suschkewitsch, Uber die endlichen Gruppen ohne das Gesetz der eindeutigen Unkehrbarkeit, Math. Ann. 99 (1928), 30-50.

46. P. S. Venkatesan, On decompositions of semigroups with zero, Math. Z. 92 (1966), 164-174.

47. R. J. Warne, Extension of completely 0-simple semigroups by completely 0-simple semigroups, Proc. Amer. Math. Soc. 17 (1966), 524-526.

48. M. Yamada, Regular semigroups whose idempotents satisfy permutation identities, Pacific J. Math. 21 (1967), 371-392.

49. H. R. Krishna Yengar, Semilattices of bisimple inverse semigroups, Semigroup Forum 2 (1971), 44-48.

50. Y. Zalcstein, Locally testable semigroups, Semigroup Forum 5 (1973), 216-227.

Dienst Hogere Meetkunde, Rijksuniversiteit Gent, KrijgslaAn 281, B-9000 Gent, Belgium 